\title{
Simulation numérique et spécification des charges dans les trémies de silos
}

\author{
Numerical simulation and specification of loads in hoppers
}

\author{
A. KHELIL \\ GRRS IUT Département Génie Civil ${ }^{*}$ \\ J.-C. ROTH \\ LPMM, Université de Mezt *
}

Rev. Franç. Géotech. n 61, pp. 85-100 (décembre 1992)

\section{Résumé}

Cet article concerne l'étude des pressions dans les trémies de silos et constitue un complément de la simulation numérique du champ de contraintes au sein de matières granulaires stockées dans des silos cylindriques à fonds plats (1). Les actions sur les parois sont déterminées à l'aide du programme d'éléments finis "SILO " (2) (Université de Karlsruhe). L'influence du processus de remplissage ainsi que les différents paramètres géométriques des trémies et des caractéristiques mécaniques de la matière ensilée sur les variations de contraintes sont mis en évidence. La comparaison des résultats numériques et analytiques (modèles classiques et normes (3) (4) (5)), complète cette étude.

\footnotetext{
Abstract

This paper described the study of the pressures in hoppers and constituted a complement of the numerical simulation of stresses developed by granular material stored in cylindrical flat bottom silos (1). The wall forces are determined using the finite element method program "SILOS" of Karlsruhe University (2). The influence of the filling process as well as the different geometrical parameters of hoppers and mechanical characteristics of ensiled materials on the stresses variation are characterized. The comparison of the numerical and analytical (draftcodes models (3) (4) (5)) results completes this work.
}

- 54601 Villers-les-Nancy Cedex.

" lle de Saulcy, 57000 Metz. 


\section{INTRODUCTION}

La trémie constitue la partie inférieure d'un silo dont le corps de cellule vertical est de section circulaire ou polygonale. La trémie munie de l'orifice de vidange du silo, de forme conique, pyramidale ou constituée de deux plans inclinés permet une vidange ordonnée du silo. Au cours du remplissage d'un silo, les efforts exercés par la matière sur les parois verticales sont établies à partir de l'équilibre d'une tranche horizontale de matière ensilée (théories de JANSSEN, AIRY...). Dans la trémie, l'équilibre de la matière pose un problème particulier dans la mesure où la section d'étude est variable en fonction de la profondeur, imposant ainsi à l'équation différentielle de JANSSEN des hypothèses supplémentaires. Plusieurs théories (3) (4) (5) respectant globalement l'équilibre de la matière dans la trémie ont été développées en exploitant les hypothèses simplificatrices de distribution uniforme ou non de la pression verticale sur la couche de matière étudiée (fig. 1). Les études expérimentales ont montré toutefois les limites de ces théories.

Nous nous intéressons à la détermination des efforts exercés par la matière sur les parois de trémies en phase statique sans tenir compte de la surchage de la partie supérieure à la trémie (fût du silo). Les paramètres fondamentaux intervenant dans la simulation sont l'inclinaison de la paroi et sa rugosité.

Ce travail a pour but d'établir le rôle des différents paramètres dans l'évolution des pressions en paroi et à l'intérieur de la matière à partir de la simulation numérique utilisant les lois de comportement de LADE et de KOLYMBAS (1) (2).
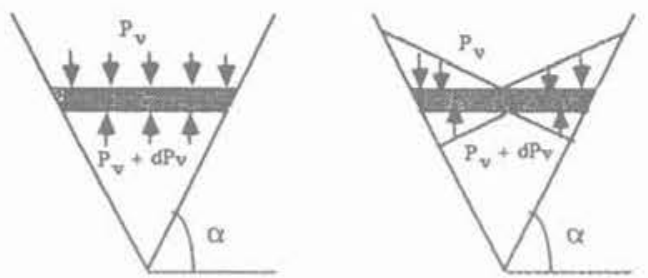

Fig. 1. - Distribution de la contrainte verticale sur une couche de matière ensilée.

Fig. 1. - Vertical stresses distribution on an elemental slice of material in a hopper.

\section{MODÈLE ANALYTIQUE DE CALCUL DES PRESSIONS}

Les formulations analytiques utilisées dans P'étude comparative sont celles de MOTZKUS, DABROWSKI, LENCZNER et WALKER. Toutes ces méthodes utilisent la théorie de JANSSEN en tenant compte de la variabilité du rayon hydraulique en fonction de la hauteur. Une couche de matière d'épaisseur dz en équilibre peut être décomposée en deux éléments en équilibre de section rectangulaire et triangulaire.

La projection des forces traduisant l'équilibre d'une tranche horizontale de matière suivant l'axe vertical s'écrit :

$$
\begin{aligned}
& P_{v} A(z)-\left(P_{v}+d_{v}\right) A(z) \\
& -\tau U(z) d z+\gamma A(z) d z=0 \\
& \left(\gamma d z-d P_{v}\right) A(z)-\tau U(z) d z=0
\end{aligned}
$$
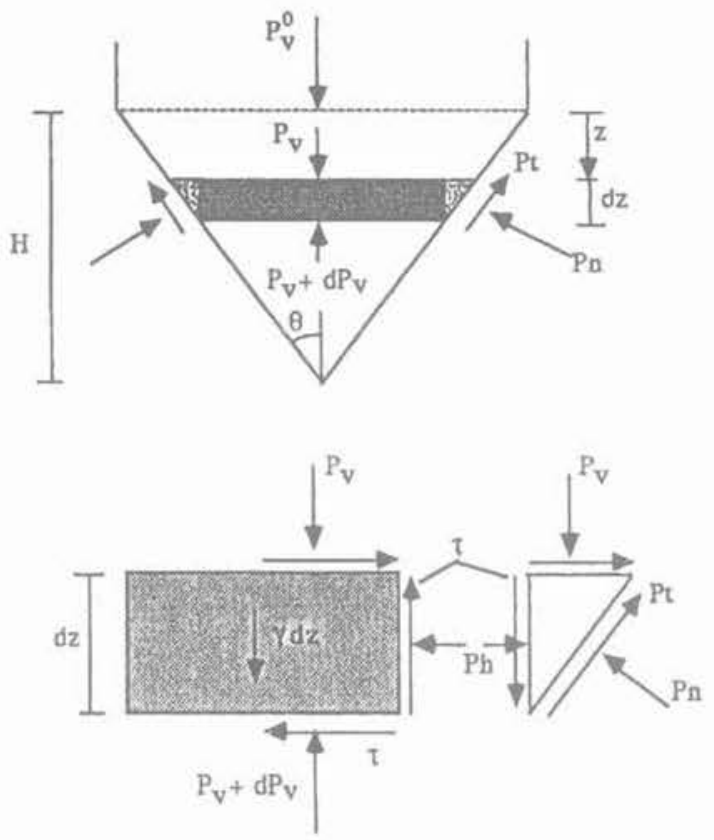

Fig. 2. - Equilibre d'une tranche de matière à l'intérieur de la trémie.

Fig. 2. - Force balance on an elemental slice of material in a hopper

$A(z)$ et $U(z)$ sont respectivement l'aire et le périmètre de la couche de matière située à la profondeur $z$ de la trémie. Pour une trémie conique on peut exprimer $A(z)$ et $U(z)$ en fonction de l'aire $A_{0}$ et le périmètre $\mathrm{U}_{0}$ de la couche située à $\mathrm{z}=0$.

$$
A(z)=A_{0}\left(\frac{H-z}{H}\right)^{2}
$$

et :

$$
\mathrm{U}(\mathrm{z})=\mathrm{U}_{0}\left(\frac{\mathrm{H}-\mathrm{z}}{\mathrm{H}}\right)
$$

En divisant l'équation (2) par $A(z) d z$, on obtient :

$$
\frac{\mathrm{dP}_{\mathrm{v}}}{\mathrm{dz}}+\frac{\mathrm{U}_{\mathrm{o}}}{\mathrm{A}_{\mathrm{o}}}\left(\frac{\mathrm{H}}{\mathrm{H}-\mathrm{z}}\right) \tau-\gamma=0
$$

Avec l'introduction du paramètre adimensionnel $\mathrm{B}=\mathrm{U}_{\mathrm{o}} \mathrm{H} \tau /\left(\mathrm{A}_{\mathrm{o}} \mathrm{P}_{\mathrm{v}}\right)$, l'équation différentielle prend la forme définitive suivante:

$$
\frac{d P_{v}}{d z}+\frac{B}{H-z} P_{v}-\gamma=0
$$

La solution de cette équation est :

$$
P_{v}=\frac{\gamma H}{1-B}\left(\xi^{B}-\zeta\right)+P_{v}^{o} \zeta^{B}
$$

avec :

$$
\zeta=\frac{H-z}{H}
$$

avec $\mathrm{P}_{\mathrm{v}}^{\mathrm{O}}$ égal à zéro si on ne tient pas compte de la surcharge de la partie supérieure à la trémie. 
On en déduit les valeurs de $\mathrm{P}_{\mathrm{h}}$ et $r$, sachant que :

$$
\mathrm{P}_{\mathrm{h}}=k \mathrm{P}_{\mathrm{v}} \text { et } \tau=k \mu \mathrm{P}_{\mathrm{v}}
$$

$\mu=\tan (\phi)$ avec $\phi:$ angle de frottement interne de la matière ensilée.

Pour le coefficient de pression $\mathrm{k}$, plusieurs formules sont proposées et ce sont souvent les expressions de $k$ qui différencient les théories conduisant aux modèles analytiques.

Les pressions normales $\left(P_{n}\right)$ et tangentielles $\left(P_{t}\right)$ à la paroi sont obtenues grâce aux relations d'équilibre sur l'élément triangulaire le long de la paroi:

$$
\begin{aligned}
& P_{n}=P_{v}\left((\sin \theta)^{2}+k(\cos \theta)^{2}+\frac{\tau}{P_{v}} \sin 2 \theta\right) \\
& \left.P_{t}=P_{v}\left(\frac{1-k}{2} \sin 2 \theta+\frac{\tau}{P_{v}} \cos 2 \theta\right)\right)
\end{aligned}
$$

\section{SIMULATION NUMÉRIQUE}

La simulation développée à partir des lois de comportement élastoplastique de LADE et hypoélastique de KOLYMBAS (1), à laide du programme "SILO * par éléments finis permet l'analyse des contraintes à partir de la variation:

- du processus de remplissage ;

- de la géométrie de la trémie (inclinaison de la paroi et taille de l'orifice de vidange) ;

- des propriétés mécaniques du matériau (frottement en paroi, poids propre...).

Si le modèle élastoplastique de LADE est bien connu, il est intéressant par contre de préciser les caractéristiques de la loi de KOLYMBAS.

Loi de comportement de KOLYMBAS

Cette loi propose une nouvelle approche du comportement des milieux granulaires. Elle repose sur une seule relation tensorielle liant le taux de contrainte aux contraintes et au taux de déformation. Cette équation tient compte des déformations non élastiques des sols (plastiques ou non réversibles) mais contrairement à la théorie de la plasticité elle ne fait pas de distinction entre les déformations élastiques et les déformations plastiques; elle n'utilise pas non plus la notion de surface d'écoulement.

Pour des raisons d'objectivité matérielle, la loi de comportement de KOLYMBAS utilise la dérivée de JAUMANN objective $\tau$ du tenseur des contraintes de CAUCHY T définie par :

$$
\tau=\frac{\mathrm{dT}}{\mathrm{dt}}-\Omega \mathrm{T}+\mathrm{T} \Omega
$$

où le tenseur des rotations $\Omega$ constitue la partie antisymétrique de la décomposition du tenseur gradient de vitesse (grad V) en partie symétrique et antisymétrique grad $V=D+\Omega$. La loi de KOLYMBAS s'écrit :

$$
\begin{aligned}
& \tau=C_{1} \frac{\mathrm{TD}+\mathrm{DT}}{2}+\mathrm{C}_{2} \operatorname{tr}(\mathrm{TD}) \mathrm{I} \\
& +\mathrm{C}_{3} \mathrm{~T} \sqrt{\operatorname{tr}(\mathrm{D})^{2}}+\mathrm{C}_{4} \mathrm{~T}^{2} \frac{\sqrt{\operatorname{tr}(\mathrm{D})^{2}}}{\operatorname{tr}(\mathrm{T})}
\end{aligned}
$$

où $C_{1}, C_{2}, C_{3}$ et $C_{4}$ sont des paramètres liés au matériau et tr désigne la trace du tenseur correspondant.

Les deux premiers termes sont linéaires par rapport à $\mathrm{D}$ et décrivent un comportement hypoélastique. La dissipation interne et les déformations irréversibles sont traduites par le troisième et quatrième terme qui sont non linéaires.

Détermination des paramètres de la loi

La loi dépend de quatre paramètres, séparément sans signification particulière, mais permettant de décrire ensemble un phénomène donné. On peut les déterminer à partir d'un seul essai en compression triaxiale classique. La courbe contraintes-déformations permet de calculer la pente à l'origine $\mathrm{E}_{\mathrm{o}}$ et l'angle de frottement interne $\Phi$; la courbe variation de volumedéformation permet de calculer langle de dilatance à l'état limite $\beta$. Cependant pour un milieu granulaire KOLYMBAS fixe $\beta$ égal à $-45^{\circ}$, permettant ainsi d'obtenir simplement les valeurs de certaines variables puis les paramètres du modèle à partir des courbes suivantes:

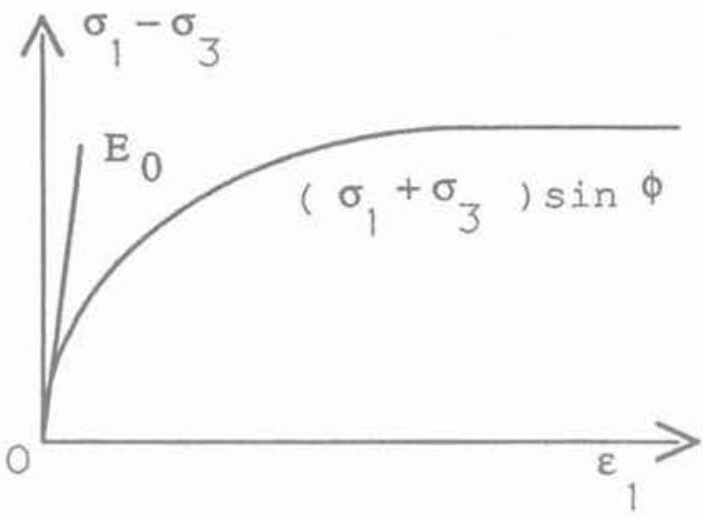

Fig. 3a. - Courbe contrainte-déformation. Fig. 3a. - Stress-strain curve.

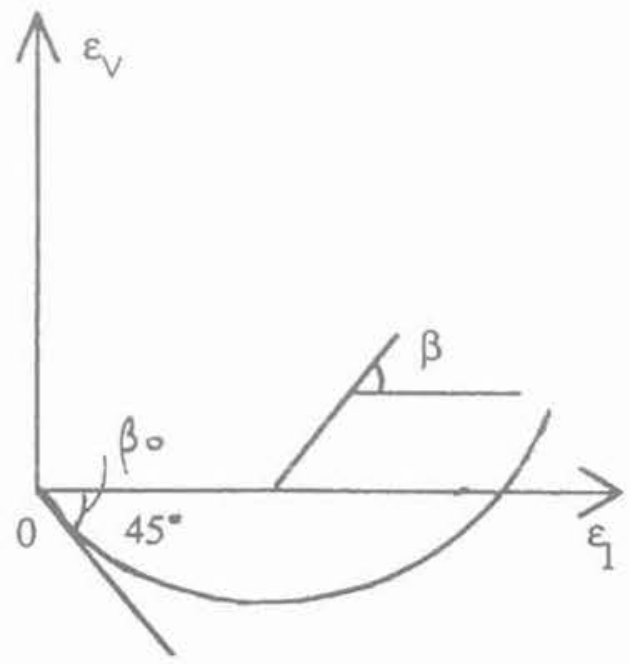

Fig. $3 b,-$ Courbe variation de volume-déformation. Fig. 3b. - Volumetric-strain curve. 


$$
\begin{gathered}
\sin \Phi=\max \frac{\sigma_{1}-\sigma_{3}}{\sigma_{1}+\sigma_{3}} ; a=\frac{1+\sin \Phi}{1-\sin \Phi} ; \\
\mathrm{bo}=\frac{1}{2}\left(1+\operatorname{tg} \beta_{0}\right)=0 ; \mathrm{b}=\frac{1}{2}(1+\operatorname{tg} \beta) ; \\
\mathrm{d}=\frac{\mathrm{a}-1}{\mathrm{a}+1}[(2 \mathrm{~b}-\mathrm{a})(2 \mathrm{a}+1)+3 \mathrm{ac}] \\
\beta_{0}=\operatorname{arctang} \frac{\operatorname{trD}}{\left|\mathrm{d}_{1}\right|}
\end{gathered}
$$

Dans le cas d'un essai de compression triaxiale classique, le tenseur des contraintes de CAUCHY T et le tenseur des vitesses de déformations $D$ sont définis par:

$$
\begin{aligned}
& T=\left[\begin{array}{lll}
\sigma_{1} & 0 & 0 \\
0 & \sigma_{3} & 0 \\
0 & 0 & \sigma_{3}
\end{array}\right] \\
& D=\left[\begin{array}{lll}
\mathrm{d}_{1} & 0 & 0 \\
0 & \mathrm{~d}_{3} & 0 \\
0 & 0 & \mathrm{~d}_{3}
\end{array}\right]
\end{aligned}
$$

- $\sigma_{3}$ est la pression de confinement, connue ; - $\mathrm{d}_{1}$ est donnée par la vitesse de déplacement du piston.

La loi de comportement se résume à deux équations :

$$
\begin{aligned}
& \dot{\sigma}_{1}=C_{1} d_{1} d \sigma_{1}+C_{2}\left(d_{1} d \sigma_{1}+2 d_{3} d \sigma_{3}\right) \\
& +C_{3} \sqrt{d_{1}^{2}+2 d_{3}^{2}} \sigma_{1} \\
& +C_{4} \sqrt{d_{1}^{2}+2 d_{3}^{2}} \frac{\sigma_{1}^{2}}{\sigma_{1}+2 \sigma_{3}} \\
& \dot{\sigma}_{3}=C_{1} d_{3} d \sigma_{3}+C_{2}\left(d_{1} d \sigma_{1}+2 d_{3} d \sigma_{3}\right) \\
& +C_{3} \sqrt{d_{1}^{2}+2 d_{3}^{2}} \sigma_{3} \\
& +C_{4} \sqrt{d_{1}^{2}+2 d_{3}^{2}} \frac{\sigma_{3}^{2}}{\sigma_{1}+2 \sigma_{3}}
\end{aligned}
$$

\begin{tabular}{|c|c|c|c|c|}
\hline & $\mathrm{C}_{1}$ & $\mathrm{C}_{2}$ & $\mathrm{C}_{3}$ & $\mathrm{C}_{4}$ \\
\hline Sable dense & -200 & $-47,9$ & 36,4 & $-252,65$ \\
\hline Sable meuble & $-128,21$ & 0 & 119,22 & $-357,65$ \\
\hline Blé & $-104,17$ & $-\quad 0,32$ & 96,22 & - 289,62 \\
\hline Colza & -50 & 3,72 & 52,6 & - 146,64 \\
\hline Sucre & -125 & 2,64 & 108,02 & -316 \\
\hline
\end{tabular}

L'application de ces deux relations à la courbe contrainte-déformation d'un essai triaxial permettra la détermination des quatre paramètres $C_{1}, C_{2}, C_{3}$ et $\mathrm{C}_{4}$ :

$$
\begin{aligned}
& C_{1}=\frac{E_{0}}{\sigma_{3}} ; C_{2}=C_{1} \frac{a}{b}\left(1+b-3 \frac{1+a b}{a+2}\right) ; \\
& C_{3}=-\frac{C_{1}}{c d}\left((2 b-a)(a+b)+3 a c \frac{a b+1}{a+2}\right) ; \\
& C_{4}=3\left(C_{2}-C_{3}\right) .
\end{aligned}
$$

Paramètres de la loi de KOLYMBAS
Tableau 1. - Valeurs des paramètres. Table 1 - Parameter values.

3.1. Simulation du processus de remplissage La simulation est réalisée avec deux trémies cylindriques d'inclinaisons $40^{\circ}$ et $70^{\circ}$ (fig. 3), successivement remplies en une seule fois, puis en dix étapes avec du sable dense en utilisant la loi de comportement de KOLYMBAS.

La simulation du remplissage est effectuée par deux procédés différents : le premier consiste à imposer le poids propre en plusieurs incréments de force, mais simultanément sur toute la hauteur de la trémie. Pour le second, plus exact mais aussi plus astreignant, le poids propre est imposé de façon incrémentale, couche après couche. Ce second procédé rend plus fidèlement compte du processus réel de remplissage. Des différences sensibles entre les deux procédés apparaissent, en particulier pour la plus grande trémie, aussi bien pour l'intensité et la direction des contraintes principales que pour les contraintes à la paroi (fig. 6, 7 et 8)

Le tassement du matériau provoque des contraintes horizontales élevées. Si, comme cela se produit pour le remplissage en une étape, on impose la même rigidité (module d'élasticité) au matériau sur toute la hauteur de la trémie, les déformations verticales seront plus impor-

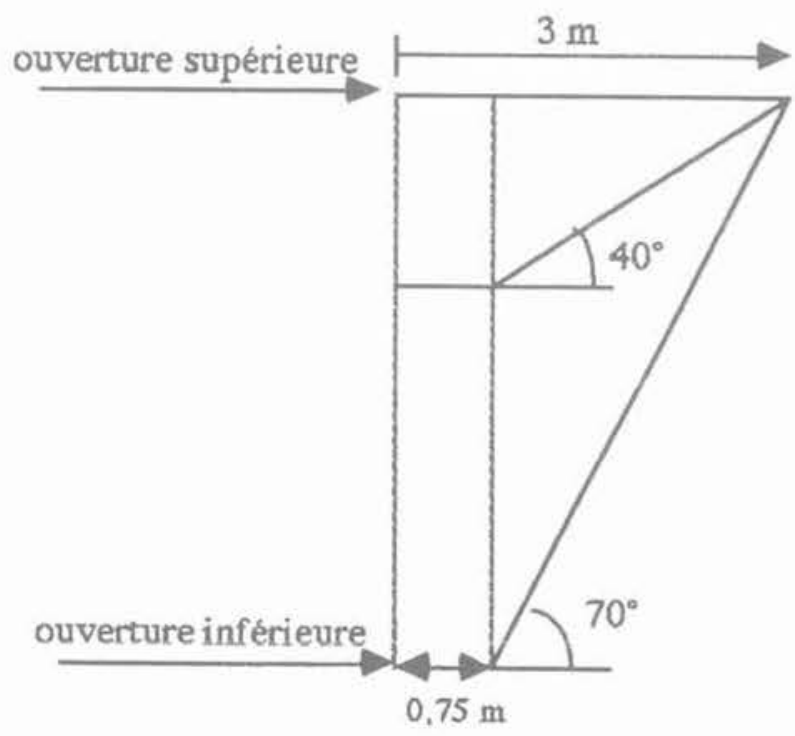

Fig. 4. - Modèles de trémies utilisés pour la simulation du remplissage. Matériau: sable dense coefficient de frottement au paroi : 0,5

Fig. 4. - Hoppers models used for the filling simulation material : dense sand: coefficient of wall friction : 0,5 . 


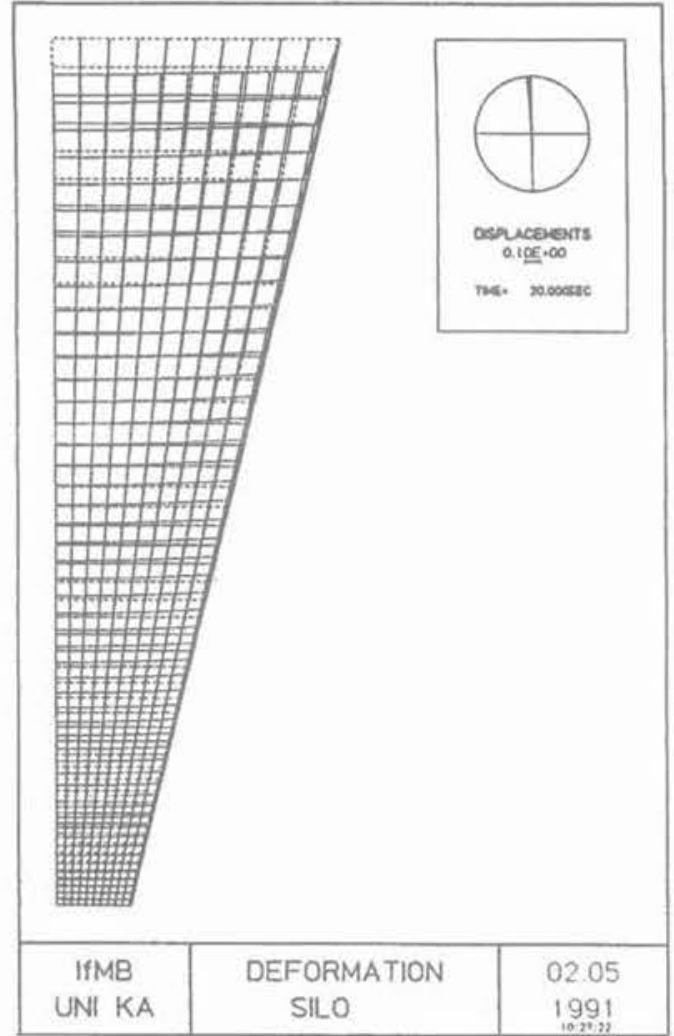

Fig. 5. - Exemple de maillage. Fig. 5. - Meshwork example.

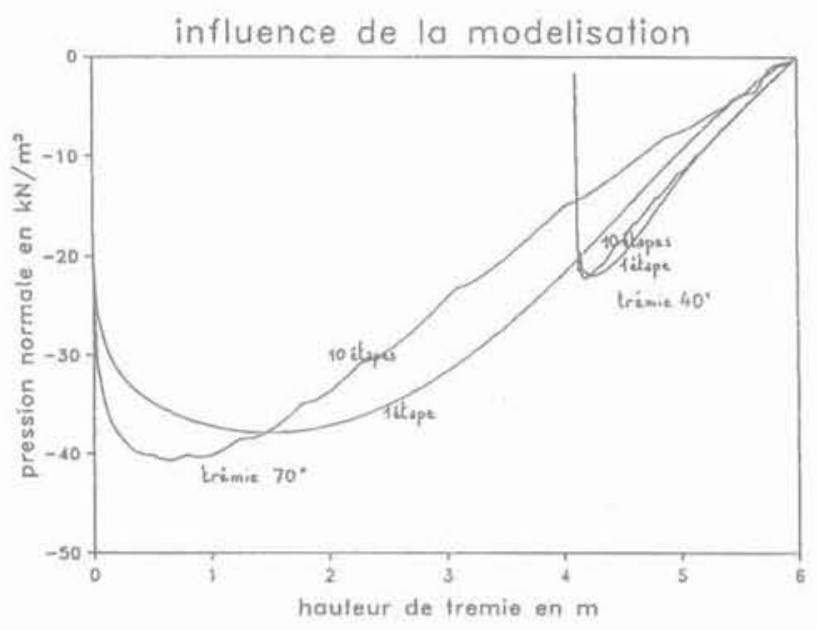

Fig. 6. - Contraintes normales à la paroi.

Fig. 6. - Normal wall stresses.

tantes dans la partie supérieure que dans la partie inférieure de la trémie. En fait, la charge est déplacée de la partie basse de la trémie vers la partie supérieure.

\subsection{Etude de l'influence de l'inclinaison} de la trémie

\subsubsection{Modèles de trémies utilisés}

La simulation est réalisée avec des trémies d'inclinaisons : $40^{\circ} ; 50^{\circ} ; 60^{\circ} ; 65^{\circ} ; 70^{\circ}$ (fig. 10), afin d'étu-

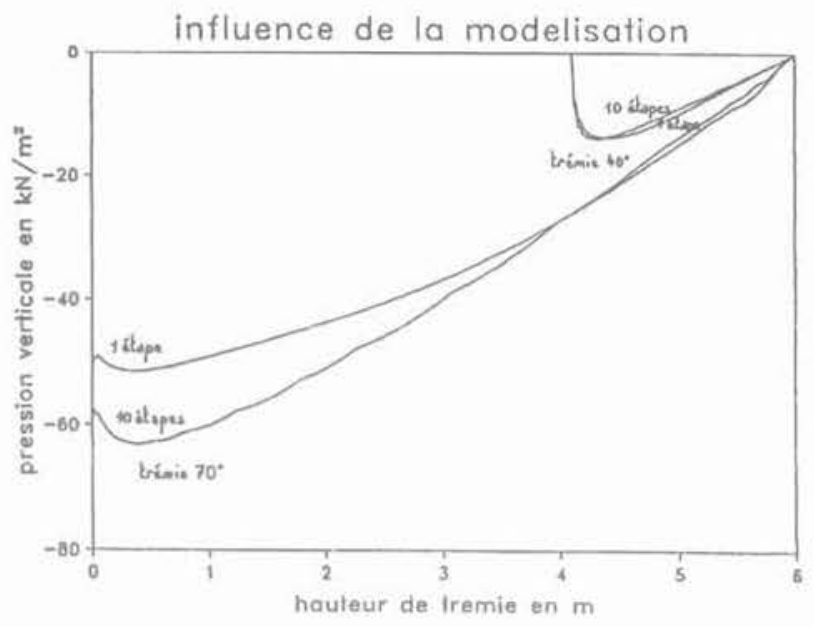

Fig. 7. - Contraintes verticales à la paroi.

Fig. 7 - Vertical stresses on the wall.

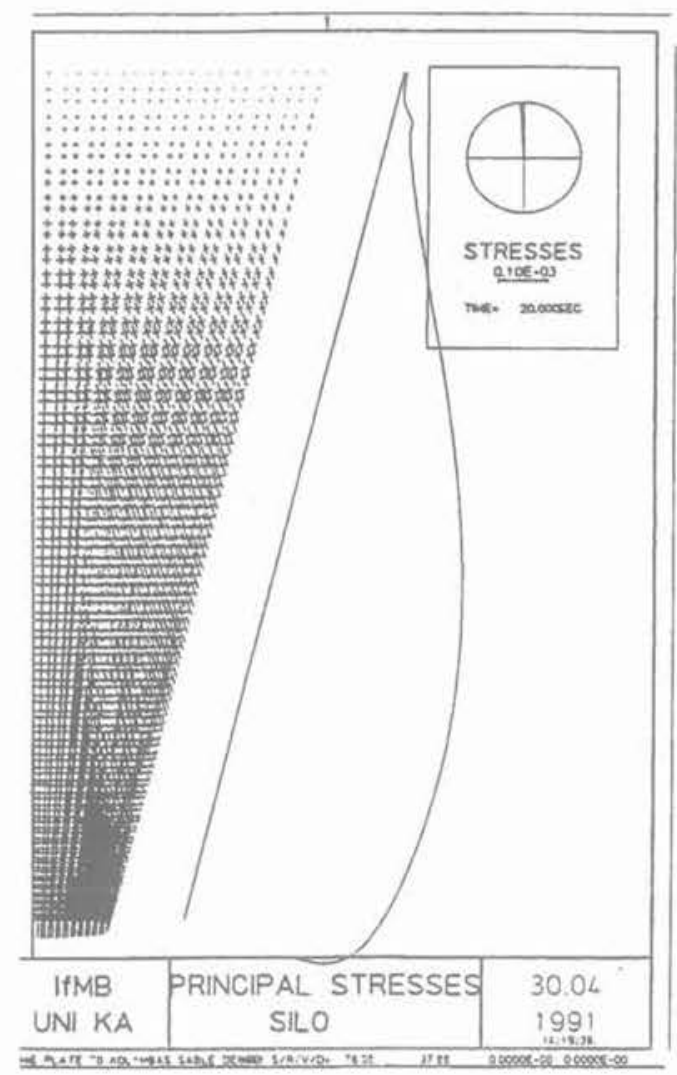

Fig. 8. - Contraintes principales dans la matière ensilée, trémie $70^{\circ}$, remplissage en une étape.

Fig. 8. - Internal principal stresses, hopper $70^{\circ}$, one filling stage.

dier l'influence de l'inclinaison de la trémie sur la distribution des contraintes. Le rapport diamètre supérieur sur le diamètre inférieur est considéré constant pour se situer dans des conditions réelles de choix de trémies pour des dimensions données de la partie cylindrique (ou fût).

Matériau : sable dense, coefficient de frottement en paroi : 0,5, loi de comportement : KOLYMBAS. 


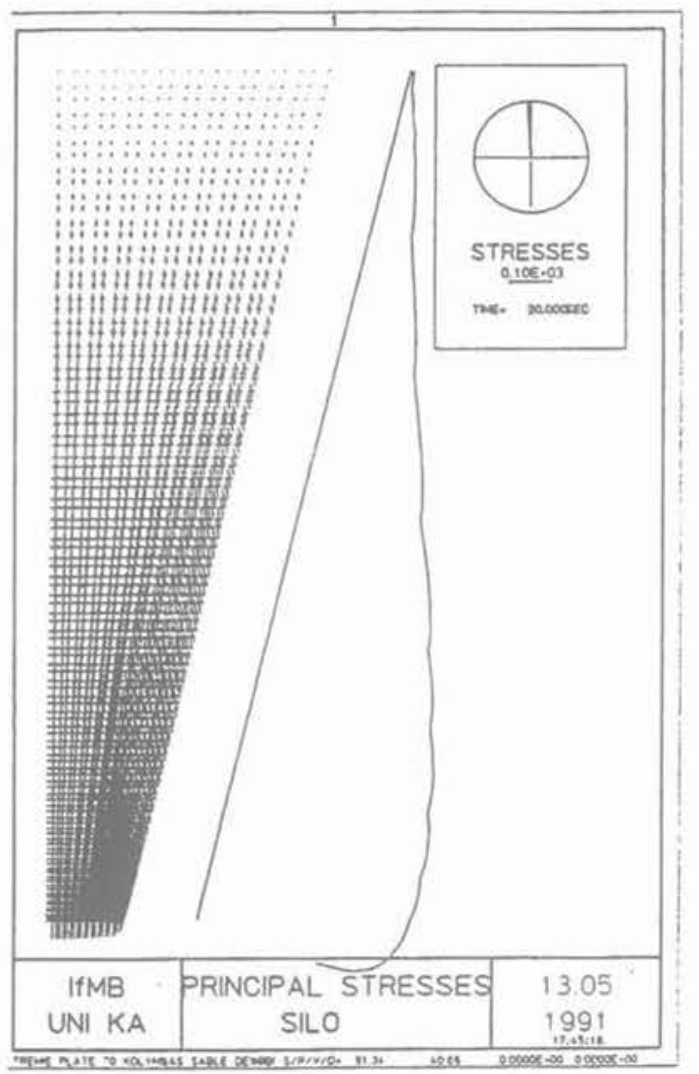

Fig. 9. - Contraintes principales dans la matière ensilée, trémie $70^{\circ}$, remplissage en 10 étapes.

Fig. 9. - International principal stresses, hopper $70^{\circ}$, 10 filling stages.

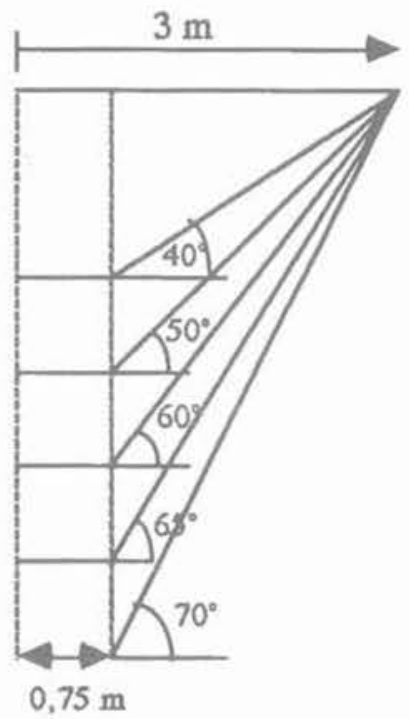

Fig. 10. - Modèles de trémies à différentes inclinaisons. Fig. 10. - Hoppers with various inclination.

\subsubsection{Distribution des contraintes à l'intérieur} de la matière ensilée

Les contraintes principales varient plus ou moins linéairement en fonction de $\mathrm{z}$ pour les trémies d'inclinaison $40^{\circ}$ et $50^{\circ}$ (fig. 11). Dans ce cas les courbes présentent un maximum près de louverture de vidan-

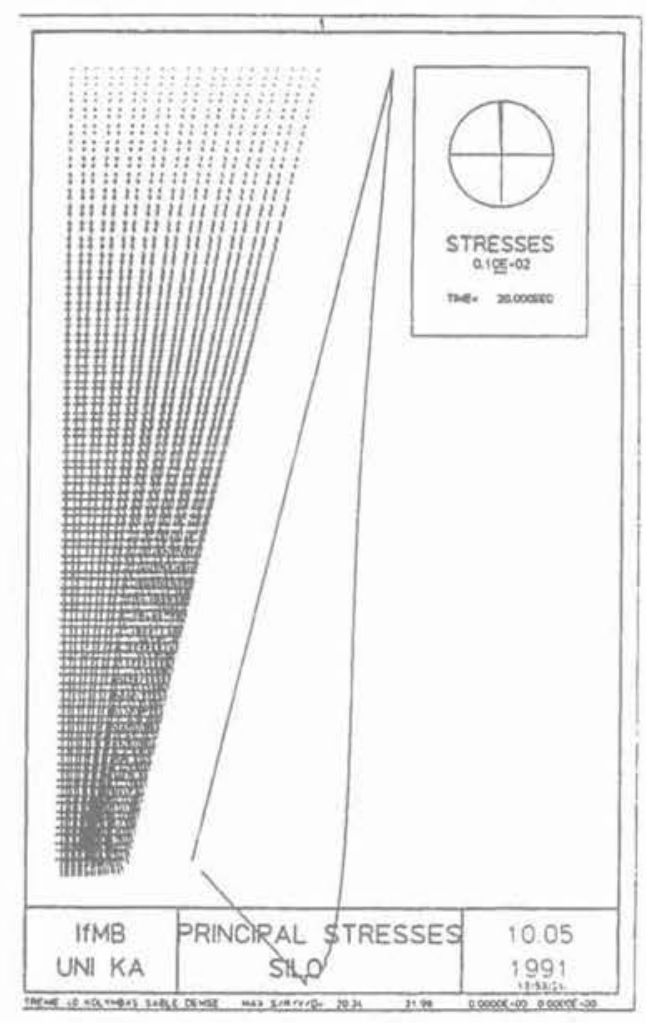

Fig. $11 a$.

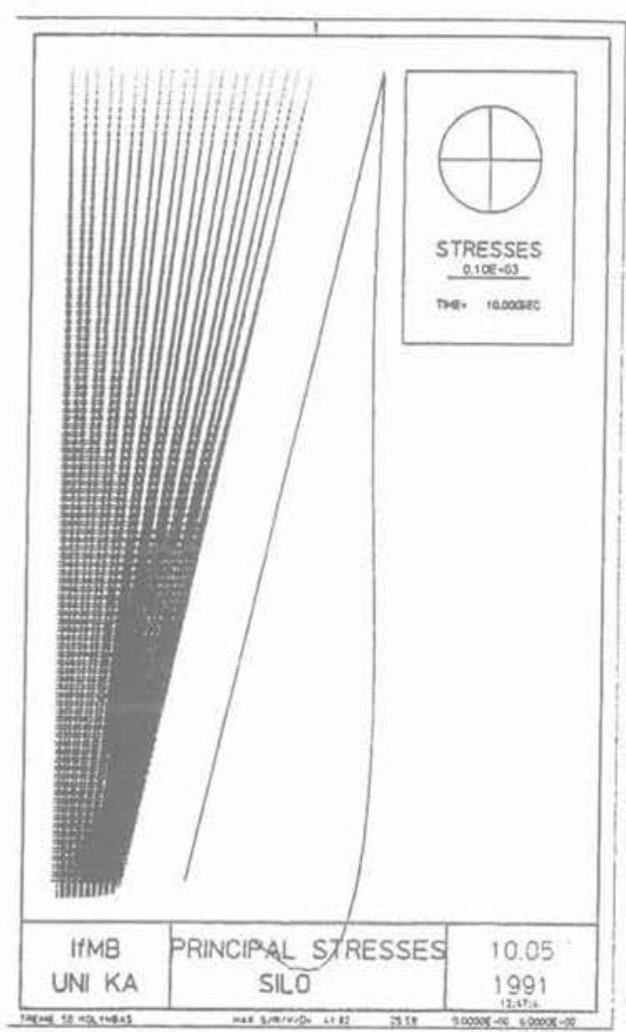

Fig. $17 \mathrm{~b}$.

Fig. 11. - Contraintes principales dans la matière ensilée. a. trémie $40^{\circ}$ - b. trémie $50^{\circ}$.

Fig. 11. - Internal principal stresses. a. hopper $40^{\circ}-b$. hopper $50^{\circ}$. 
ge. Ces courbes évoluent en fonction de l'inclinaison, passant d'une variation linéaire pour des inclinaisons faibles vers une variation non linéaire pour de fortes inclinaisons (fig. 11 et 12). La position du maximum de contrainte se déplace vers la partie supérieure de la trémie. A l'intérieur de la matière ensilée, l'inclinaison des directions principales par rapport à l'axe de symétrie vertical varie en fonction de l'angle de la trémie. Cependant l'inclinaison des directions principales par rapport à la paroi reste constante quel que soit l'angle d'inclinaison de la trémie : dans ce cas seul le frottement statique en paroi influe sur la distribution des contraintes.

\subsubsection{Pressions normale et verticale à la paroi} Le maximum de la pression normale $P_{n}$ augmente linéairement en fonction de la hauteur de la trémie. Lorsque la hauteur augmente, la pression dans la partie supérieure reste constante alors qu'elle augmente fortement dans la partie inférieure.

La position du maximum de $P_{0}$ s'éloigne de l'ouverture inférieure de la trémie, ainsi pour les petites trémies, des forces importantes sont concentrées sur une petite surface. Les variations de la pression verticale $\mathrm{P}_{\mathrm{y}}$ sont similaires. Il est donc souhaitable de construire des trémies suffisamment élancées pour ne pas surcharger la partie inférieure.

\subsection{Etude de l'influence de la taille de l'orifice de vidange}

\subsubsection{Modèles de trémies utilisés}

La simulation est réalisée avec des trémies dont le diamètre inférieur est variable, afin d'étudier l'influence de la taille de l'ouverture inférieure sur la distribution des pressions. L'inclinaison des trémies est de $70^{\circ}$ et le diamètre de l'ouverture supérieure est de $6 \mathrm{~m}$.

Rapport diamètre supérieur/diamètre inférieur : $R_{p}=0,66 ; 0,5 ; 0,33 ; 0,25$. Matériau: sable dense ; coefficient de frottement en paroi : 0.5 ; loi de comportement: KOLYMBAS.

\subsubsection{Distribution des contraintes à l'intérieur de la matière ensilée}

Lorsque l'élancement diminue, les contraintes principales diminuent et tendent à prendre les directions horizontales et verticales. Dans ce cas les parois se comportent comme un mur de soutènement et l'effet " silos » disparait.

\subsubsection{Pressions normale et verticale à la paroi} Lorsque l'ouverture diminue, la pression normale $\mathrm{P}_{\mathrm{n}}$ dans la partie supérieure reste constante, alors qu'elle augmente fortement dans la partie inférieure.

Les remarques précédentes sont également valables pour la pression verticale $P_{v}$.

\subsection{Etude de l'influence du frottement en paroi}

\subsubsection{Modèles de trémies utilisés}

La simulation est réalisée avec deux types de trémies cylindriques, en faisant varier la valeur du coefficient de frottement en paroi.

Trémie 1 : diamètre supérieur : $6 \mathrm{~m}$; diamètre inférieur: $1,5 \mathrm{~m}$; inclinaison de la paroi : $70^{\circ}$.

Trémie 2 : diamètre supérieur : $1 \mathrm{~m}$; diamètre inférieur : $0,2 \mathrm{~m}$; inclinaison de la paroi : $75^{\circ}$.
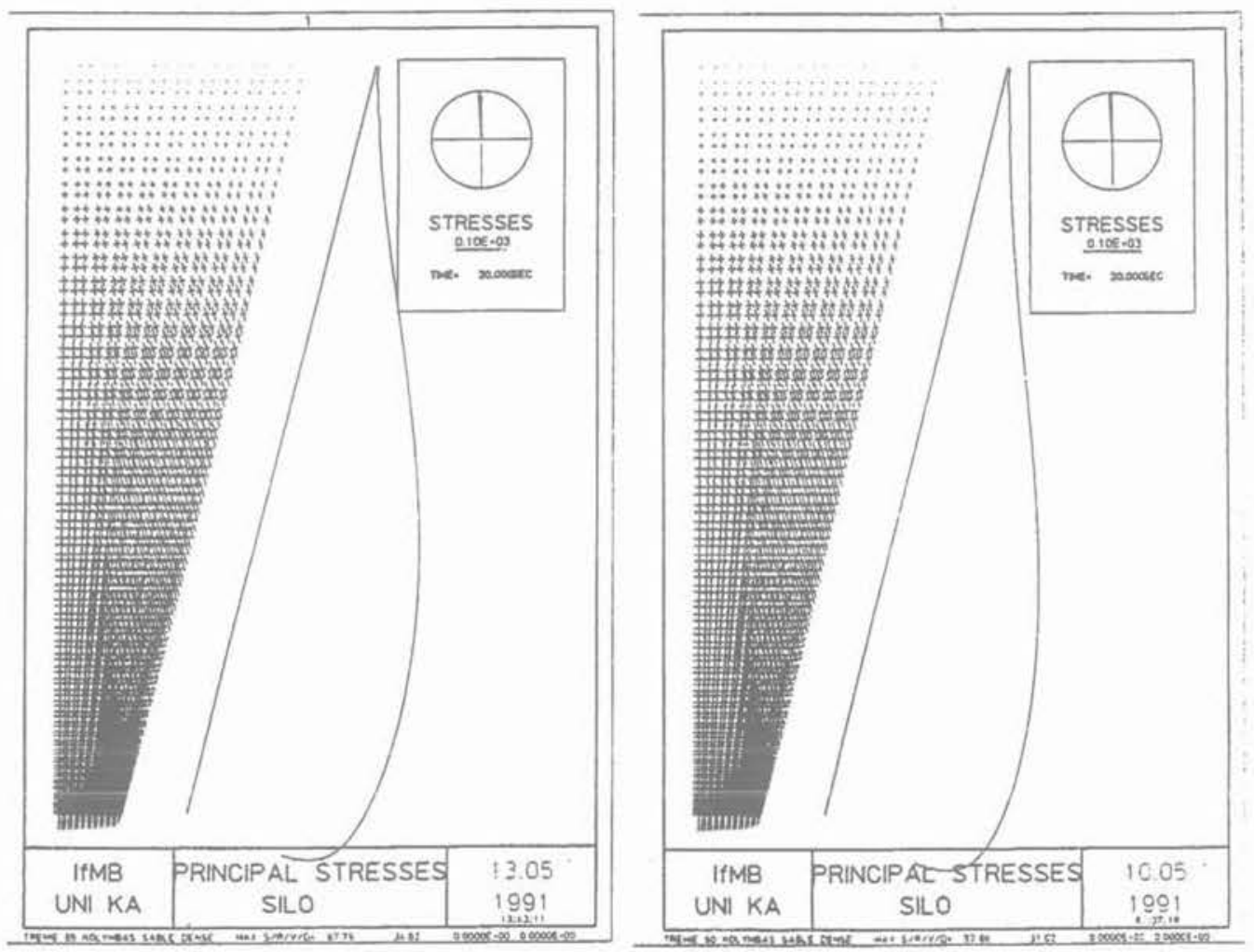

Fig. 12, - Contraintes principales dans la matière ensilée : a. trémie $60^{\circ}-b$. trémie $65^{\circ}$. Fig. 12. - Internal principal stresses: a. hopper $60^{\circ}-b$. hopper $65^{\circ}$ 


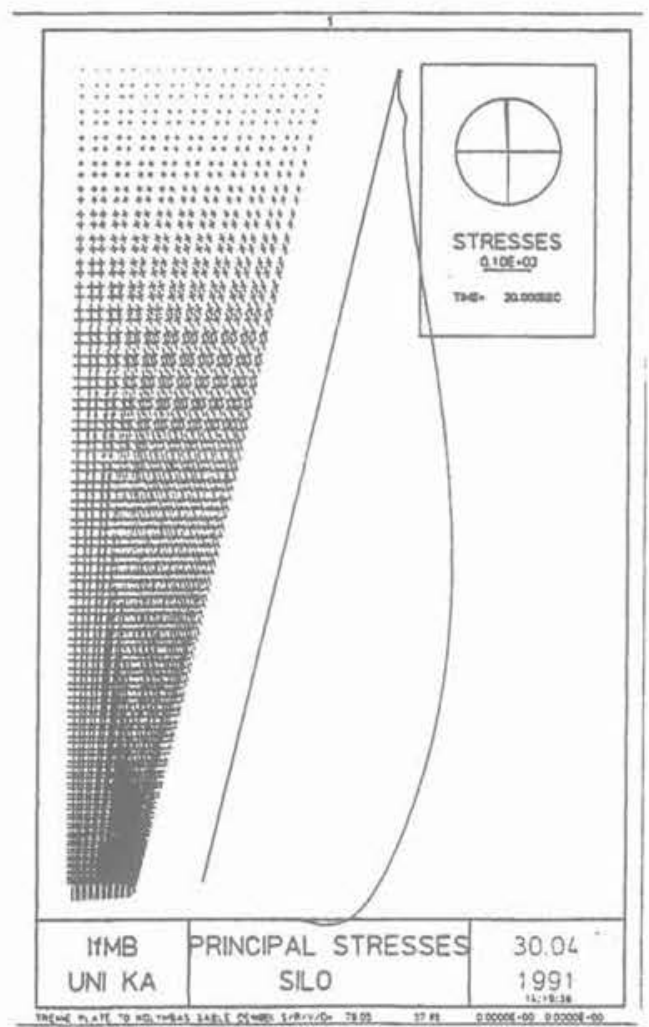

Fig. 13a. - Contraintes principales dans la matière ensilée : trémie $70^{\circ}$

Fig. 13a. - Internal principal stresses : hopper $70^{\circ}$.

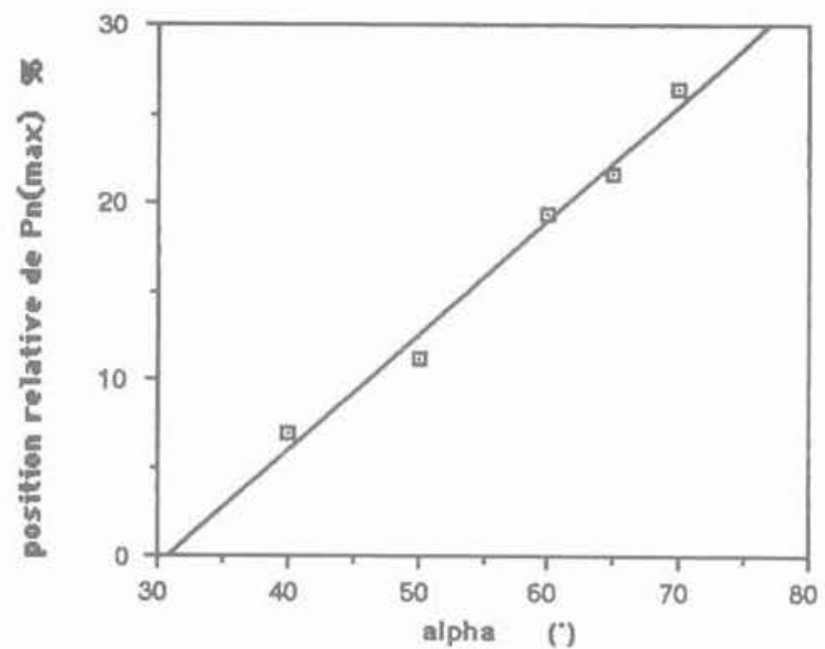

Fig. 13b. - Variation de la position relative du maximum de pression $\left(P_{n} \max \right)$ en fonction de $\alpha$.

Fig. $13 b$. - Vatiation of $P_{n}$ max relative position versus $\alpha$.

Matériau : sable dense ; coefficient de frottement en paroi : $0,1-0,2-0,3-0,4-0,5-0,6-0,7-0,8$; loi de comportement: KOLYMBAS.

\subsubsection{Distribution des contraintes à l'intérieur de la matière ensilée}

Pour un coefficient de frottement en paroi très faible (parois lisses), les contraintes principales mineures et

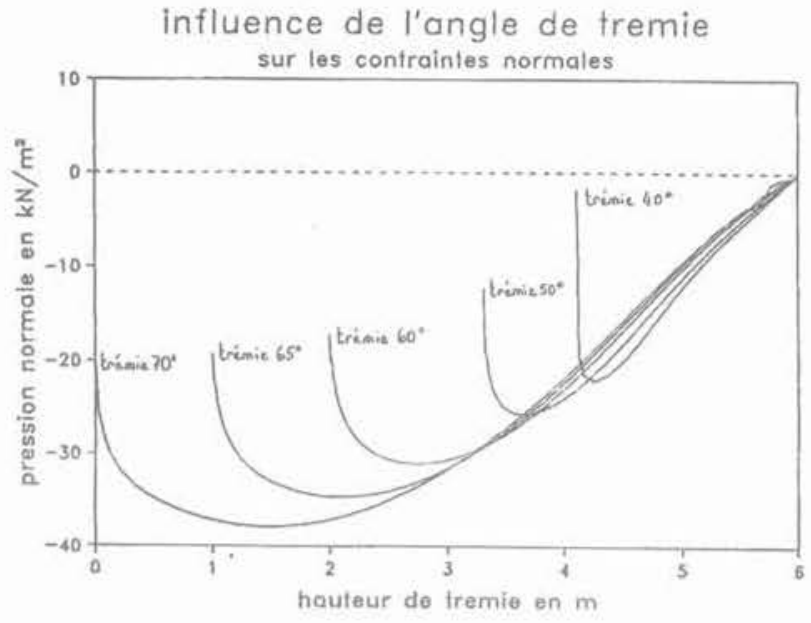

Fig. 14. - Contraintes normales à la paroi.

Fig. 14. - Normal wall stresses.

influence de l'angle de tremie

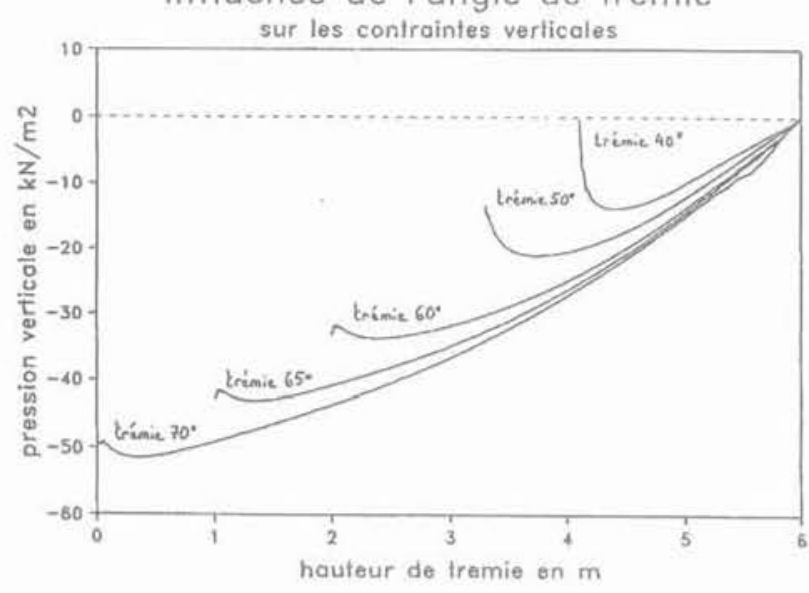

Fig. 15. - Contraintes verticales à la paroi.

Fig. 15. - Vertical stresses on the wall.

influence du rapport des diametres d'ouvertures sur les contraintes normales

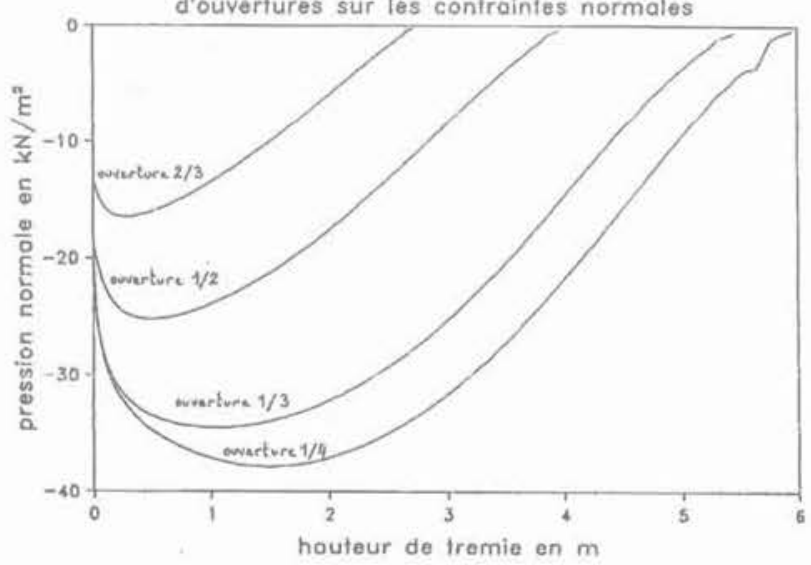

Fig. 16. - Contraintes normales.

Fig. 16. - Normal stresses distribution. 


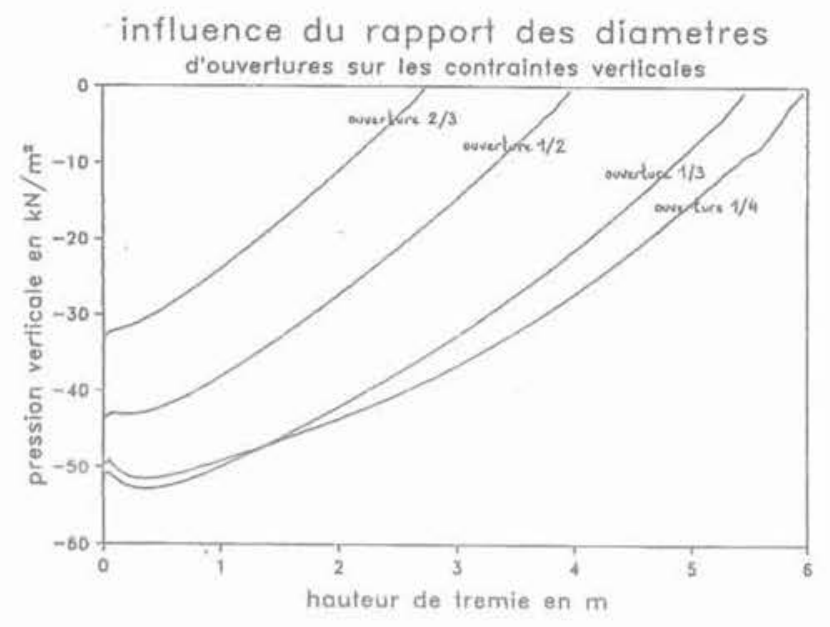

Fig, 17. - Contraintes verticales.

Fig. 17. - Vertical stresses distribution

majeures, sont respectivement horizontales et verticales, dans la partie haute de la trémie. Les contraintes majeures à une profondeur donnée s'inclinent jusqu'à devenir parallèles à la paroi dans le fond de la trémie. Lorsque le coefficient de frottement en paroi $\mu$ augmente, les contraintes principales varient aussi bien en intensité qu'en direction, en fonction de la distance à l'axe vertical de la trémie.

\subsubsection{Pressions normale et verticale à la paroi}

Le coefficient de frottement joue un rôle prépondérant dans la transmission des efforts en paroi. Les courbes des figures 24 et 25 montrent clairement le transfert d'une partie du poids propre vers la paroi en fonction de l'augmentation de sa rugosité.

\subsection{Etude de l'influence du poids propre}

\subsubsection{Modèles de trémies utilisés}

La simulation est réalisée pour les mêmes trémies qu'en 3.5 .1 ., en faisant varier le matériau ensilé, donc le poids propre, en utilisant les deux lois de comportement de LADE et de KOLYMBAS.

\section{Matériaux ;}

sable dense sable meuble blé

colza

sucre

$$
\begin{aligned}
& \gamma=16,31 \mathrm{kN} / \mathrm{m}^{3} \\
& \gamma=14,78 \mathrm{kN} / \mathrm{m}^{3} \\
& \gamma=8,05 \mathrm{kN} / \mathrm{m}^{3} \\
& \gamma=6,64 \mathrm{kN} / \mathrm{m}^{3} \\
& \gamma=8,81 \mathrm{kN} / \mathrm{m}^{3}
\end{aligned}
$$

Coefficient de frottement en paroi : 0,5, loi de comportement: KOLYMBAS et LADE.

\subsubsection{Distribution des contraintes}

La distribution des contraintes à l'intérieur du matériau a toujours la même forme. Pour une valeur de $\mu$ donnée, les contraintes normale et verticale à la paroi sont quasiment des fonctions linéaires de la densité. D'autres paramètres propres au matériau, tel que le frottement interne peuvent modifier la distribution des contraintes.
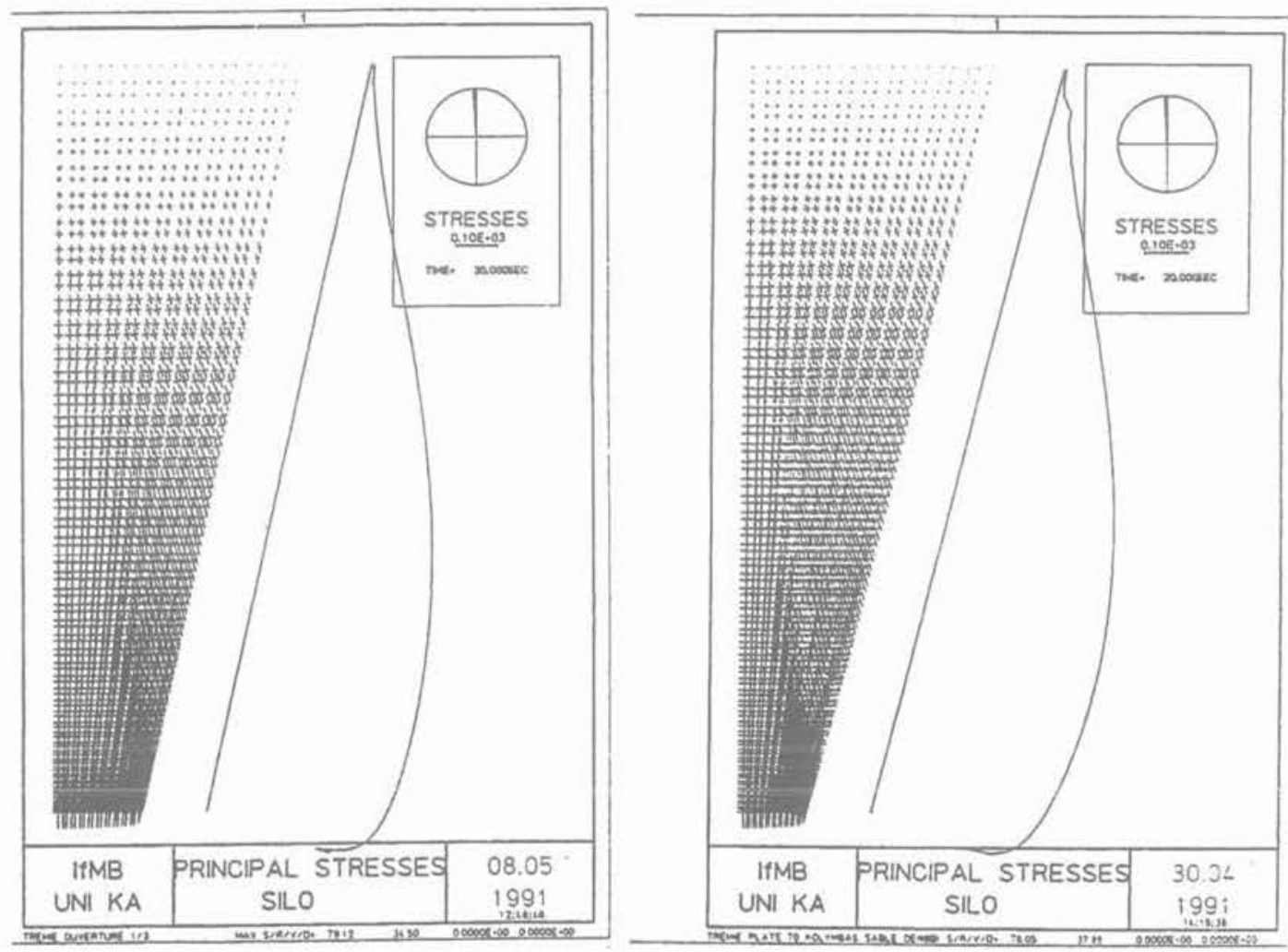

Fig. 18. - Contraintes principales: $a \cdot R_{p}=0,25-b . R_{p}=0,33$.

Fig. 18. - Principal stresses: a. $R_{p}=0,25-b . R_{p}=0,33$. 

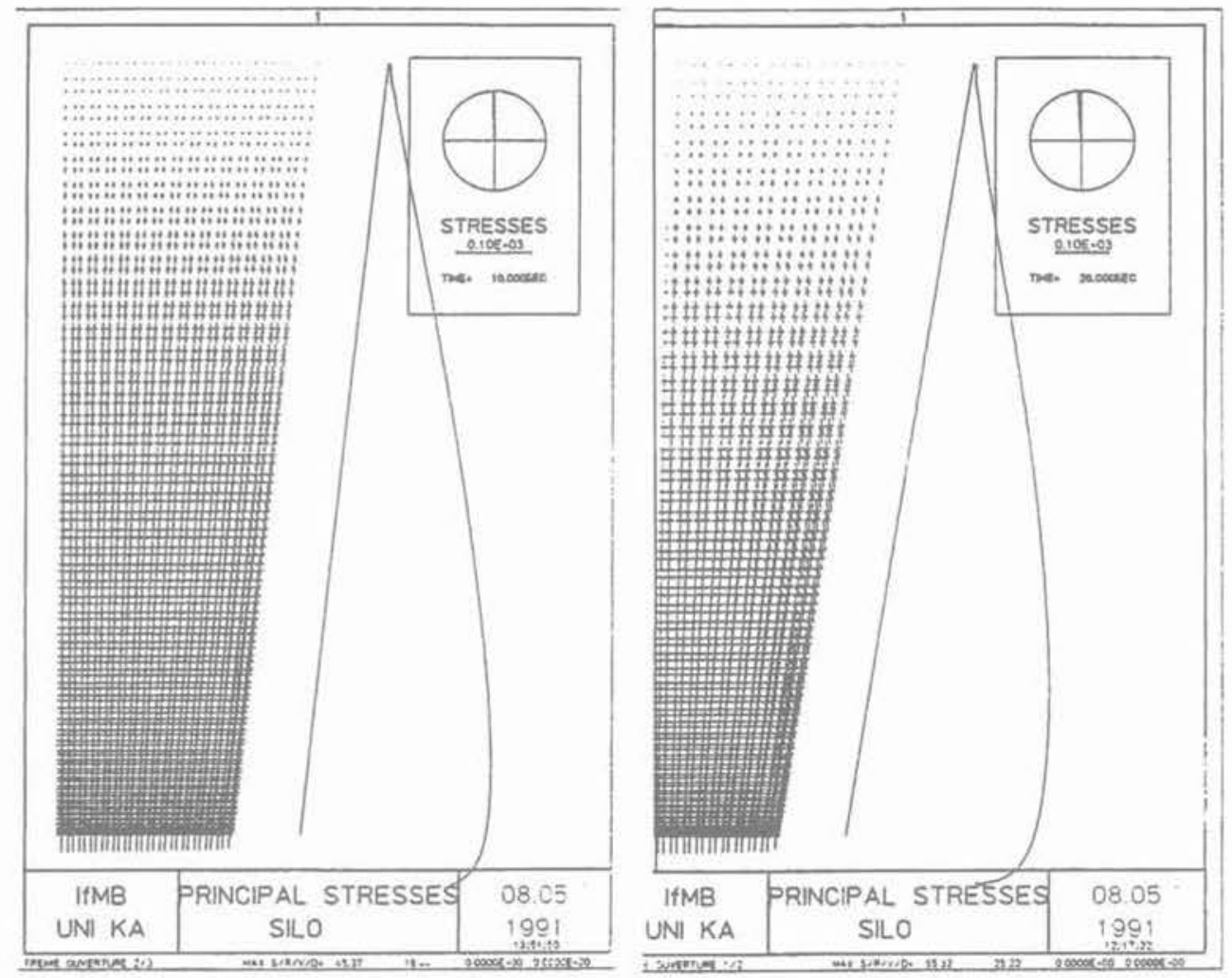

Fig. 19. - Contraintes principales ; a. $R_{p}=0,5-b . R_{p}=0,66$.

Fig. 19. - Principal stresses: $a . A_{p}=0,5-b . R_{p}=0,66$.
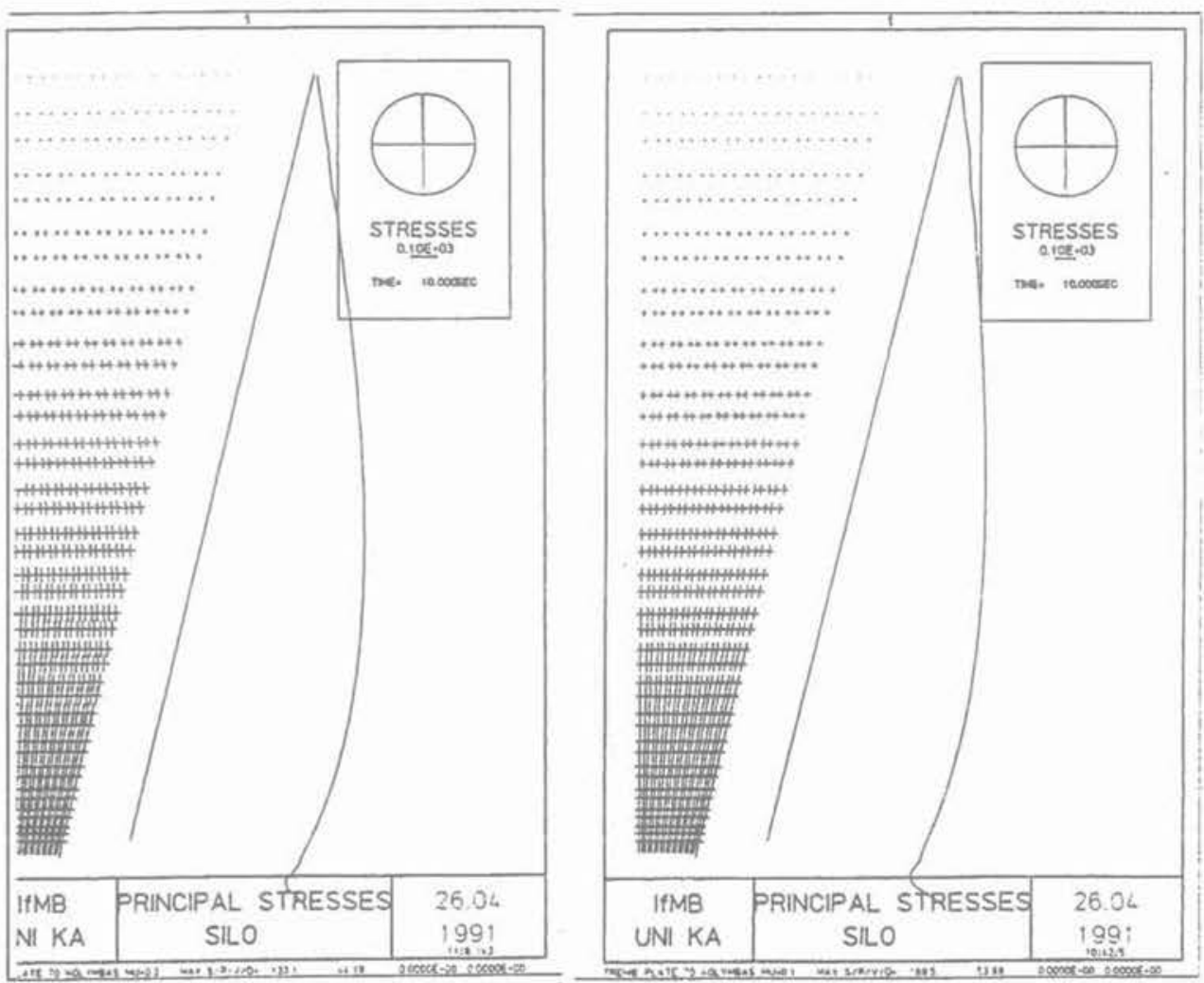

Fig. 20. - Contraintes principales: a. $\mu=0,1$ - b. $\mu=0,2$.

Fig. 20. - Principal stresses: a. $\mu=0,1-$ b. $\mu=0,2$. 

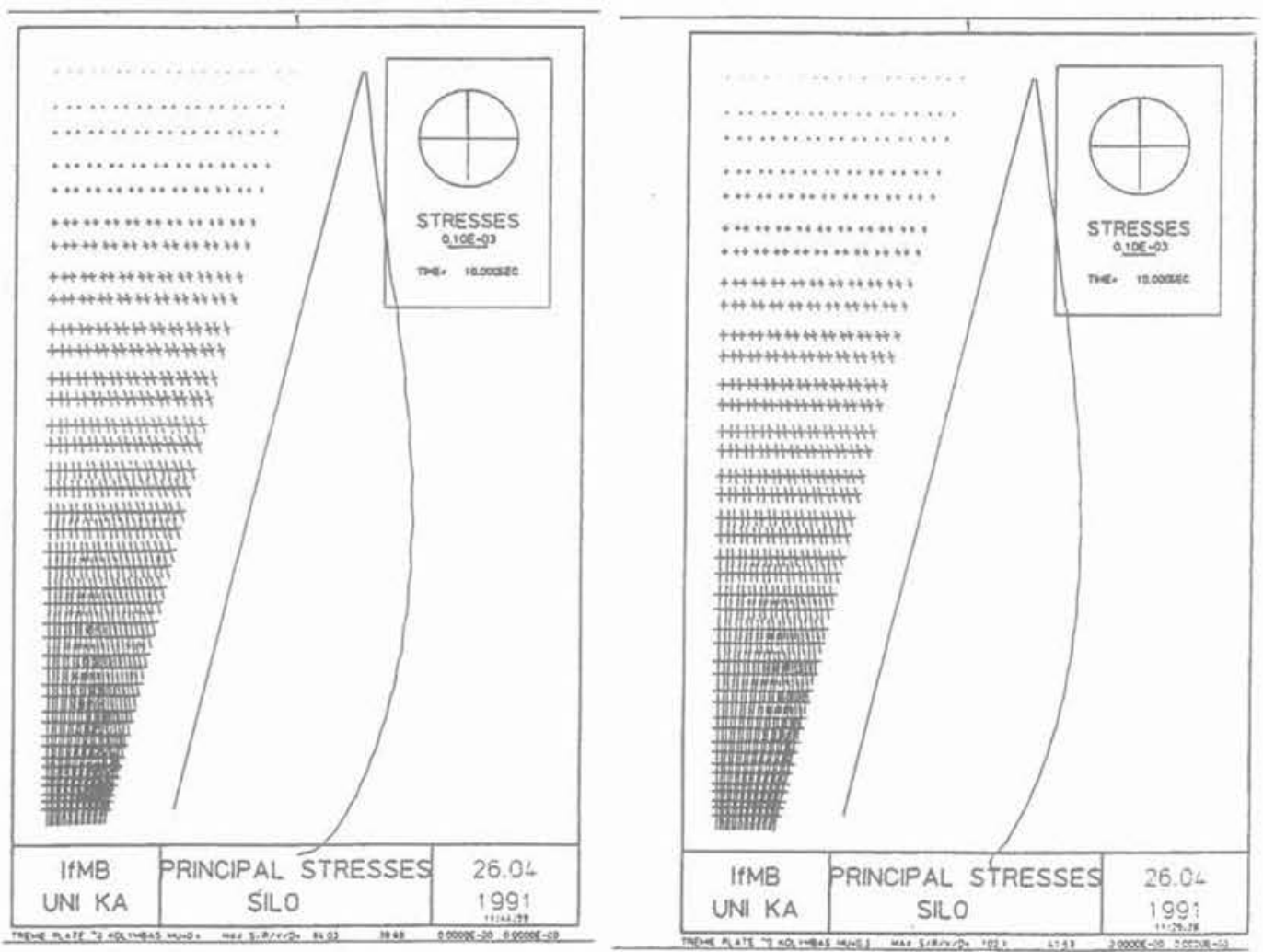

Fig. 21. - Contraintes principales: a. $\mu=0,3-b . \mu=0,4$.

Fig. 21. - Principal stresses : a. $\mu=0,3-b, \mu=0,4$
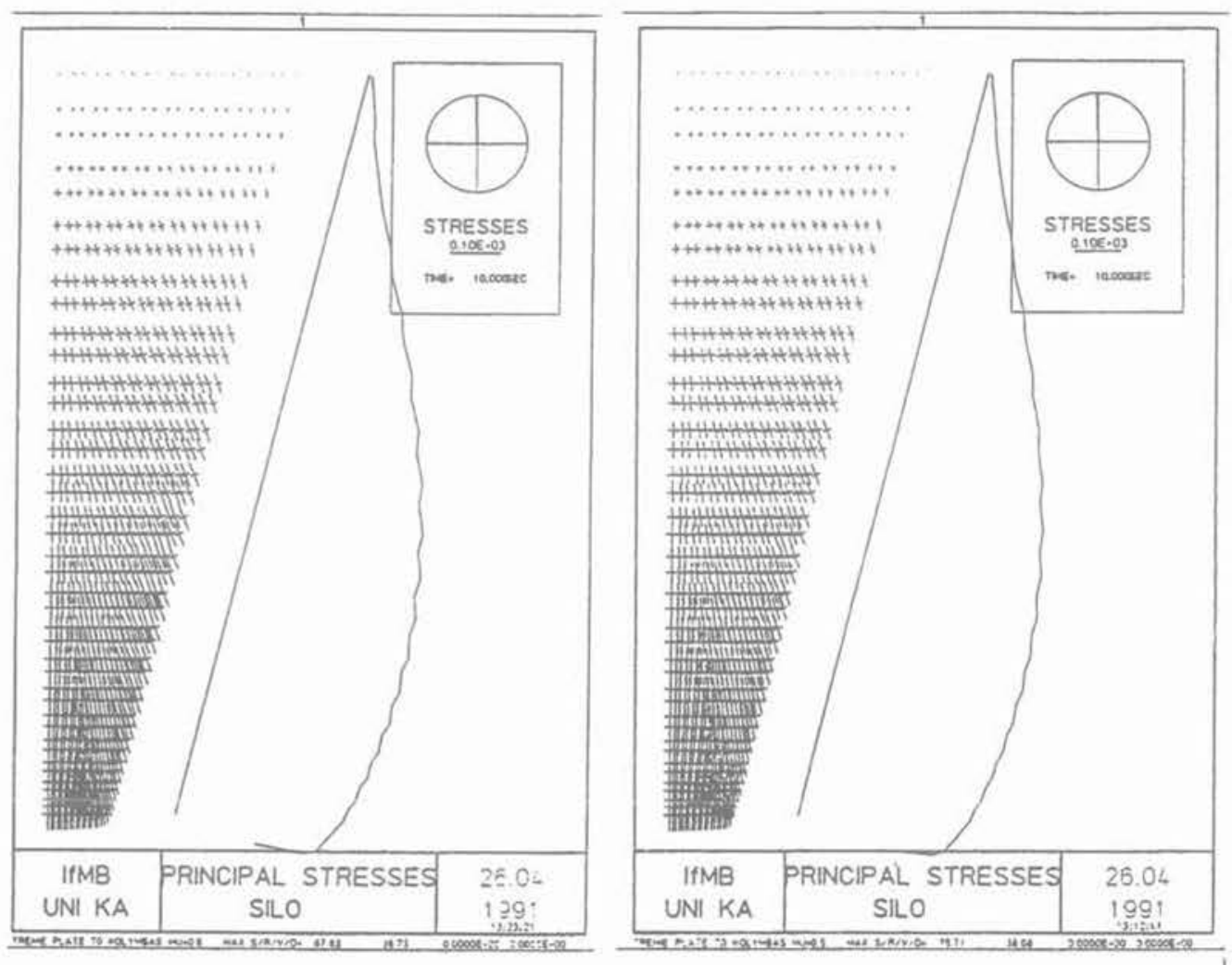

Fig. 22. - Contraintes principales: a. $\mu=0,5-b . \mu=0,6$.

Fig. 22 - Principal stresses : a. $\mu=0,5-b . \mu=0,6$. 

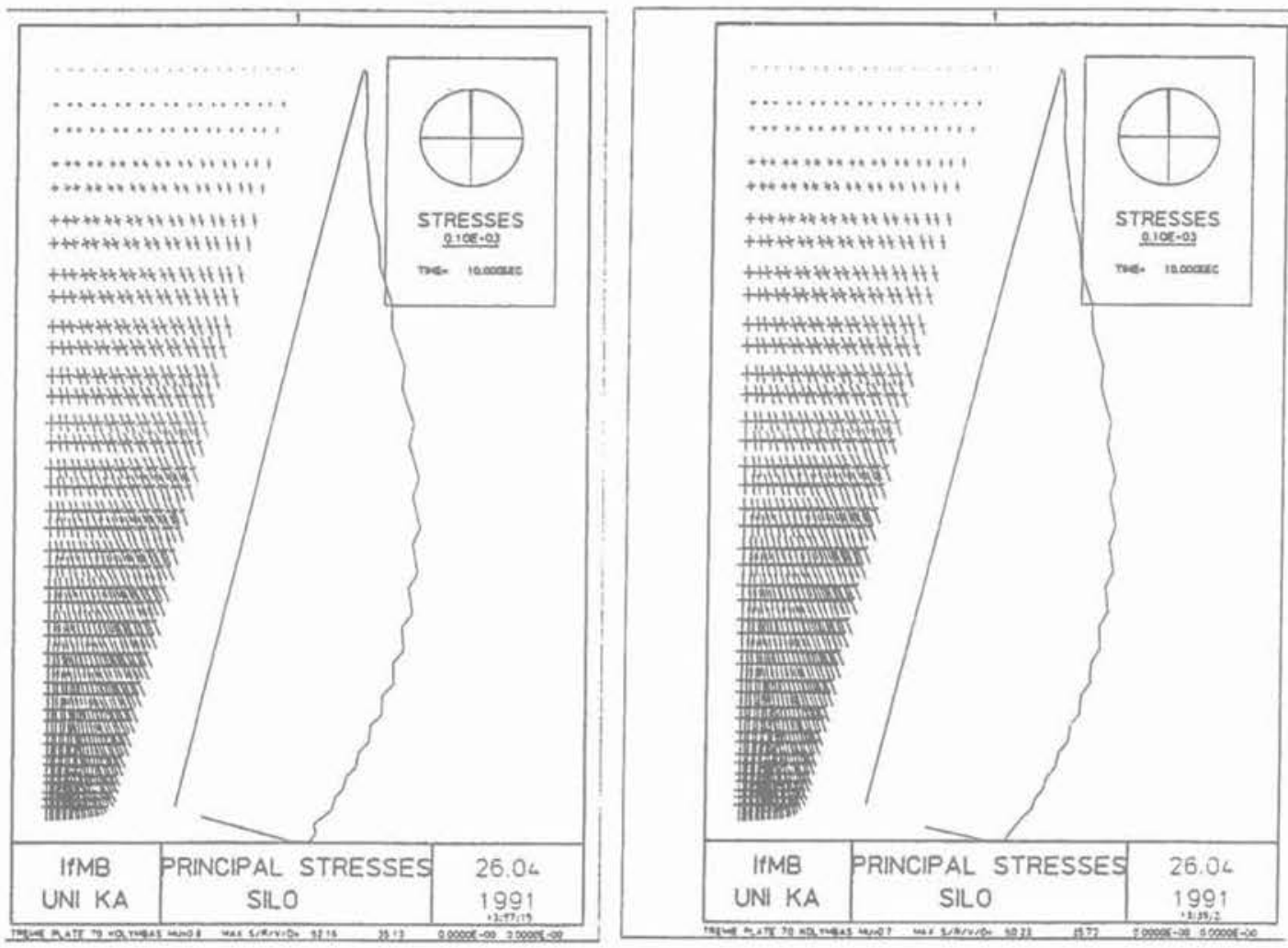

Fig. 23. - Contraintes principales : (a) $\triangleleft=0,7-(b) \triangleleft=0,8$.

Fig. 23. - Principal stresses: (a) $\triangleleft=0,7-(b) \triangleleft=0.8$

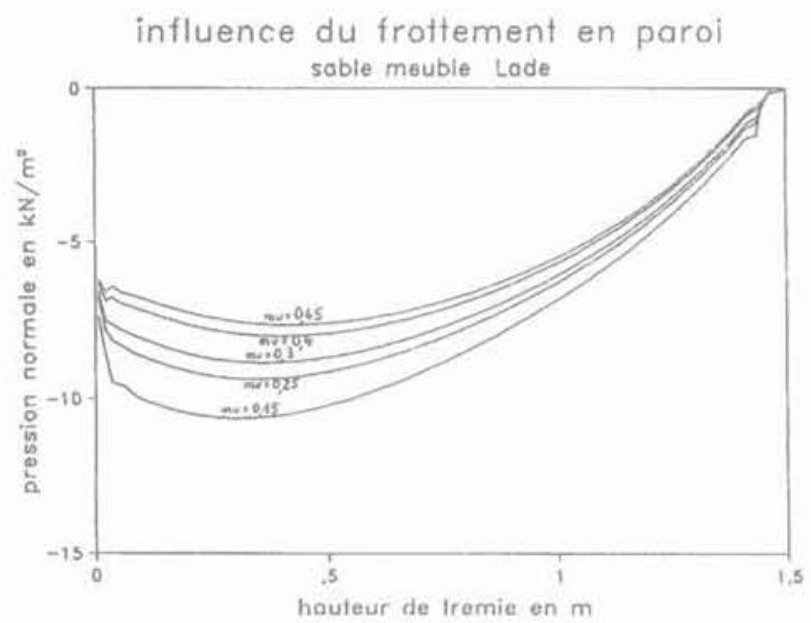

Fig. 24. - Contraintes normales: loi de comportement de LADE.

Fig. 24. - Normal wall stresses distribution LADE constitutive law.

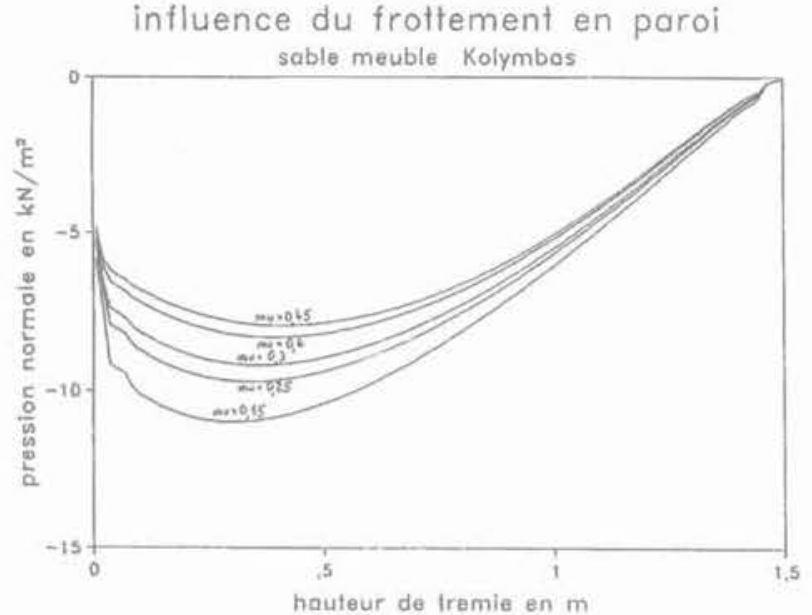

Fig. 25. - Contraintes normales : loi de comportement de KOLYMBAS.

Fig. 25. - Normal wall stresses distribution KOLYMBAS constitutive law:

\section{COMPARAISON ENTRE RÉSULTATS NUMÉRIQUES ET ANALYTIQUES}

Dans cette étude comparative, le calcul numérique est mené avec la seule loi de KOLYMBAS en utilisant les mêmes types de trémies qu'en 3.5 .1., remplies soit de sable dense soit de blé. Les modèles analytiques comparés aux résultats numériques sont ceux de LENCZNER, DABROWSKI, WALKER, MOTZKUS et la norme DIN. Les figures 30 et 31 montrent les variations de contraintes normales et verticales en fonction de la profondeur. Ces courbes sont très dispersées, toutefois la courbe de la norme DIN est proche du maximum de pression de la courbe obtenue par calcul numérique. 

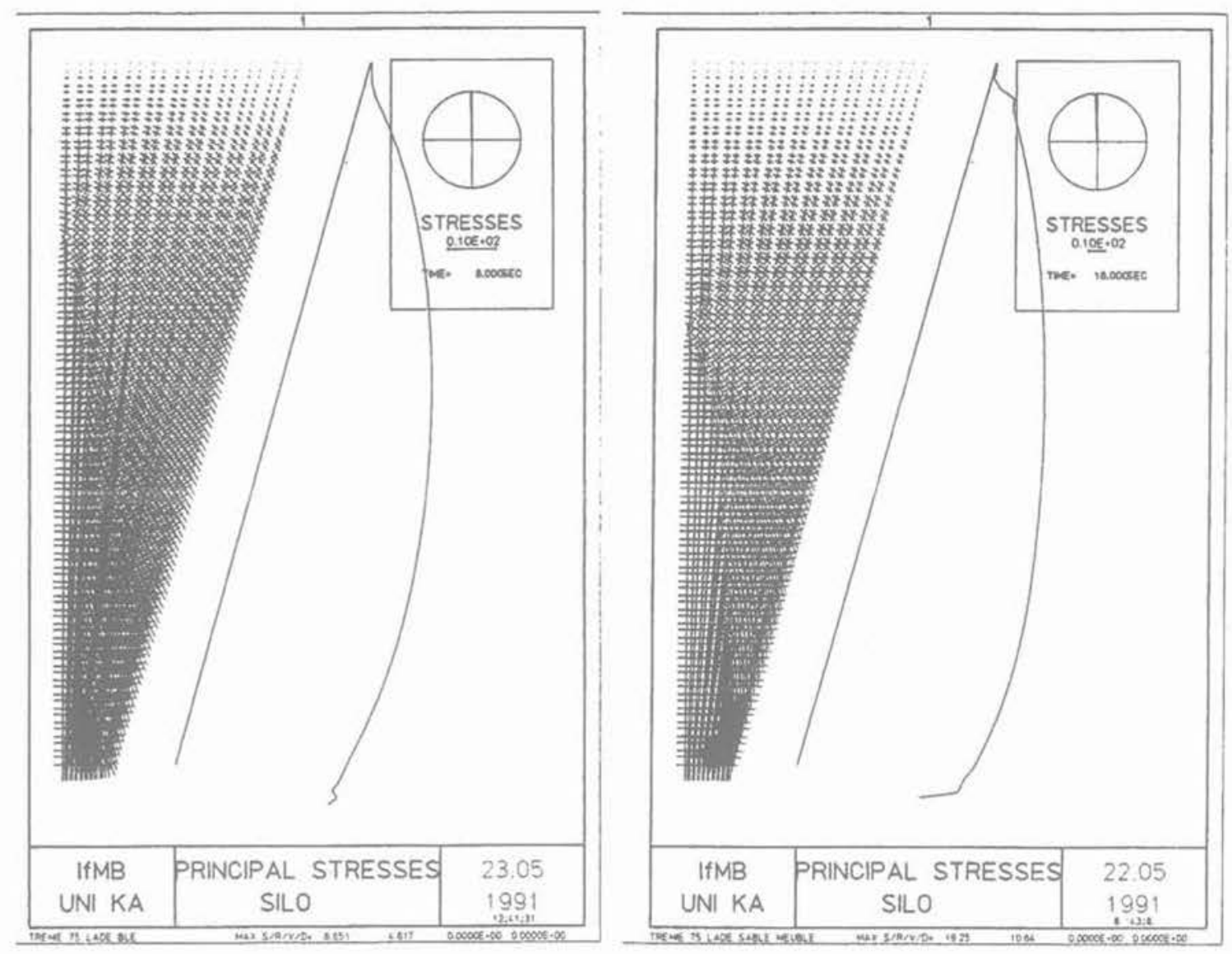

Fig. 26. - Contraintes principales : a. blé - b. sable meuble. (Loi de comportement de LADE). Fig. 26. - Principal stresses: a. wheat - b. loose sand. (LADE constitutive law).
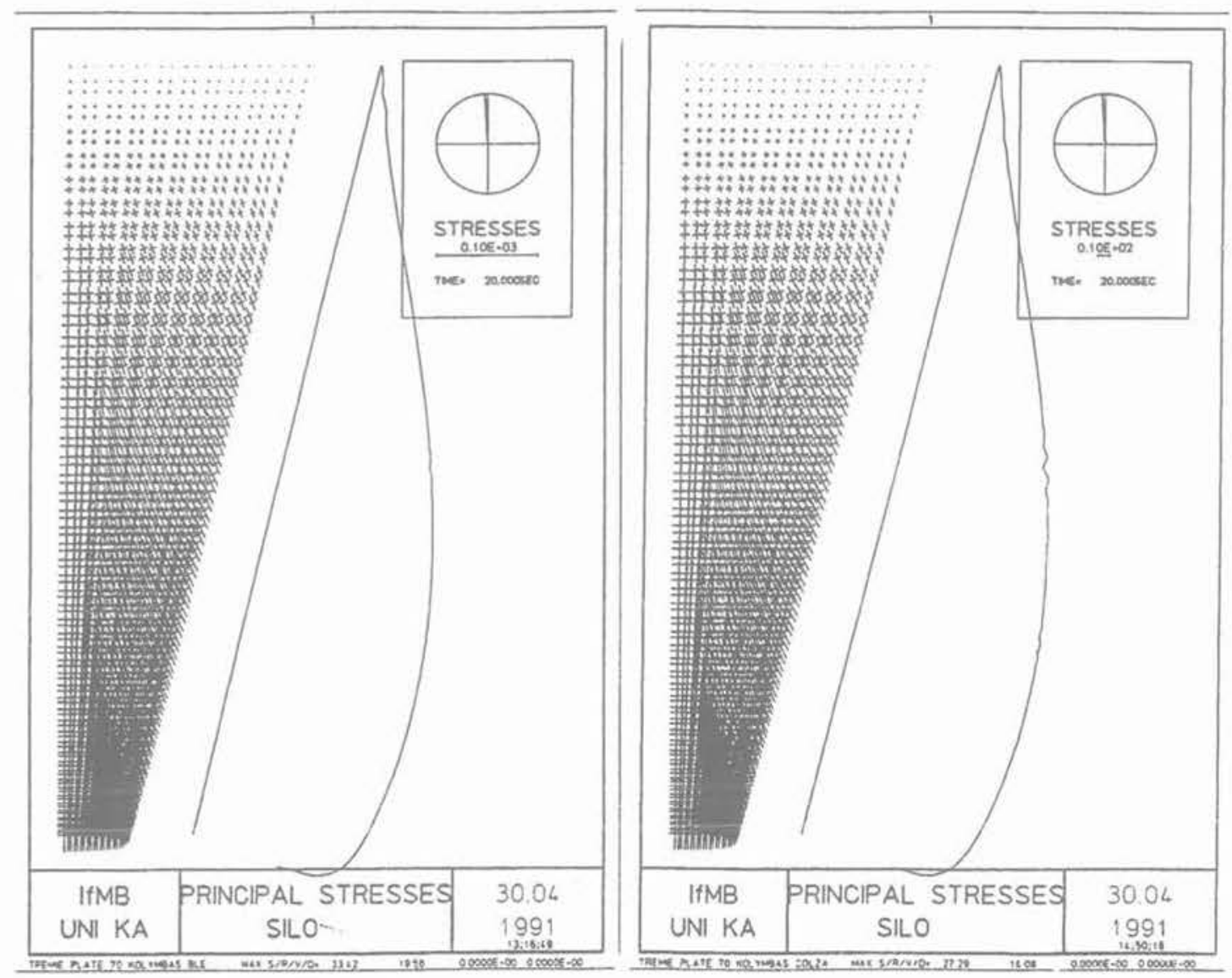

Fig. 27. - Contraintes principales: a. blé - b. colza (loi de comportement de KOLYMBAS).

Fig, 27 - Principal stresses; a, wheat - b. colsa (KOLYMBAS constitutive law). 


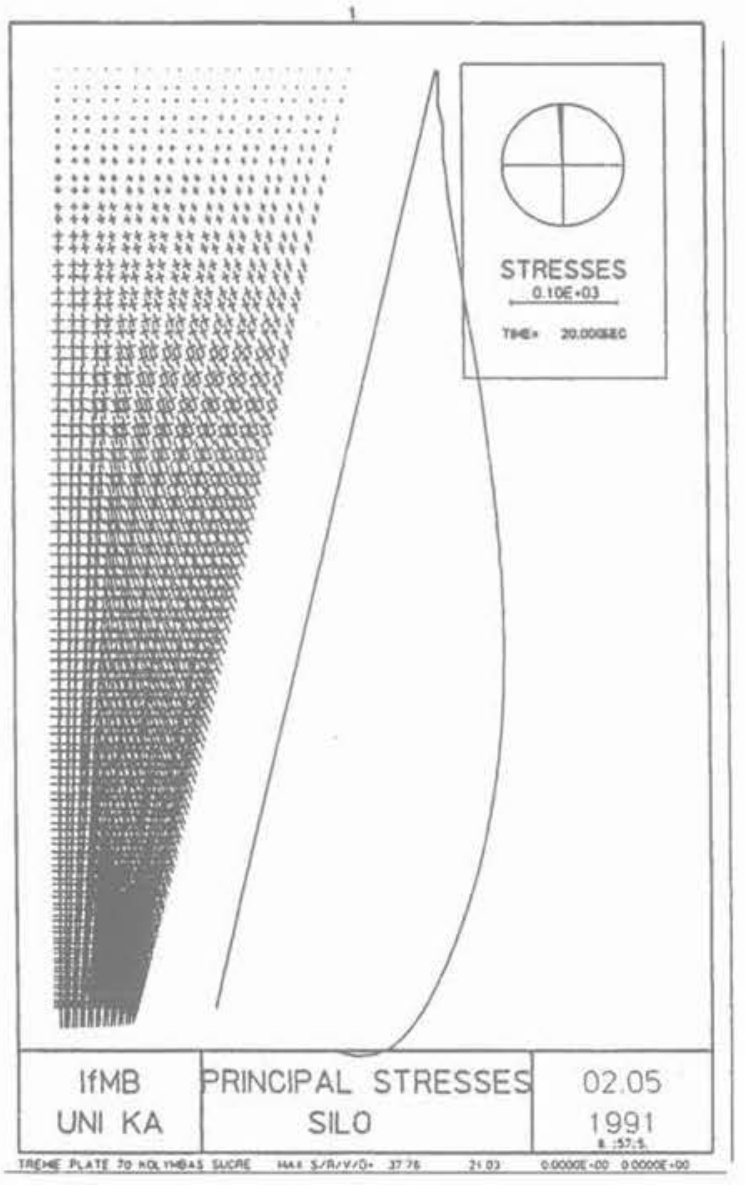

Fig. 28. - Contraintes principales : sucre (loi de comportement de KOLYMBAS). Fig. 28. - Principal stresses: sugar (KOLYMBAS constitutive law).

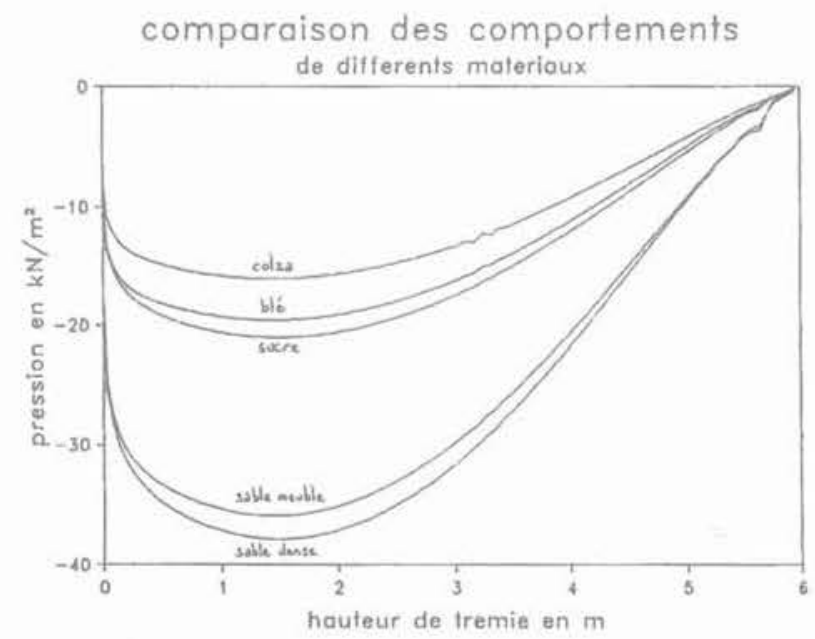

Fig. 29. - Contraintes normales à la paroi. Fig. 29. - Normal wall stresses.

\subsection{Valeur maximale de la contrainte normale à la paroi}

Pour les différentes trémies, lécart relatif entre la valeur théorique du maximum de contrainte normale
Comparaison avec les resultats theoriques sable dense

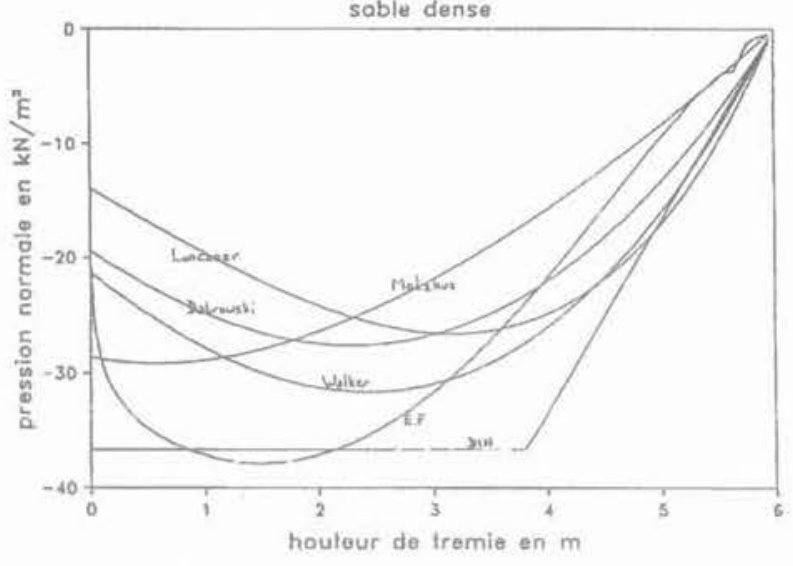

Fig. 30. - Contraintes normales à la paroi : sable dense. Fig. 30. - Normal wall stresses: dense sand.

Comparaison avec les resultats theoriques ble

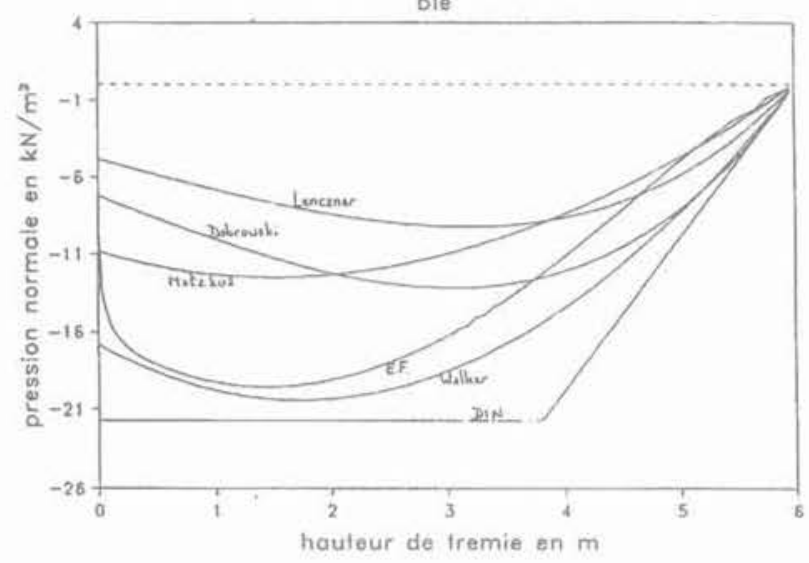

Fig. 31. - Contraintes normales à la paroi : blé. Fig. 31. - Normal wall stresses: wheat.

$\mathrm{P}_{\mathrm{n}}(\max )$ et le résultat du calcul par éléments finis est donné dans le tableau 2.

\subsection{Position du maximum de la contrainte normale à la paroi}

Pour les différentes trémies, l'écart relatif entre la valeur théorique de l'ordonnée du maximum de la contrainte normale et le résultat du calcul par éléments finis est donné dans le tableau 3.

\subsection{Valeur maximale de la contrainte verti-} cale à la paroi

Pour les différentes trémies, l'écart relatif entre la valeur théorique du maximum de la contrainte verticale et le résultat du calcul par éléments finis est donné dans le tableau 4.

\subsection{Comparaison avec les normes DIN 87 et $\mathrm{P} 22-630$}

La norme DIN donne une approximation imprécise des contraintes à la paroi de la trémie. Les valeurs de la norme sont souvent supérieures aux valeurs 
Tableau 2. - Ecart relatif entre $P_{n}(\max )$ théorique et numérique.

Table 2. - Relatif deviation between theoretical and numerical value of $P_{n}(\max )$.

\begin{tabular}{|c|c|c|c|c|c|}
\hline \multirow{2}{*}{ Trémies } & \multirow{2}{*}{ Matériaux } & \multicolumn{4}{|c|}{ Modèles analytiques } \\
\hline & & MOTZKUS & DABROWSKI & LENCZNER & WALKER \\
\hline \multirow{2}{*}{ Trémie $70^{\circ}$} & sable dense $\mu=0,5$ & $23 \%$ & $27 \%$ & $30 \%$ & $16 \%$ \\
\hline & blé $\mu=0,5$ & $36 \%$ & $30 \%$ & $53 \%$ & $-\quad 4 \%$ \\
\hline \multirow{4}{*}{ Trémie $75^{\circ}$} & sable meuble $\mu=0,25$ & $14 \%$ & $26 \%$ & $26 \%$ & $18 \%$ \\
\hline & sable meuble $=0,4$ & $32 \%$ & $-24 \%$ & $34 \%$ & $23 \%$ \\
\hline & blé $\mu=0,25$ & $34 \%$ & $-30 \%$ & $7 \%$ & $4 \%$ \\
\hline & blé $\mu=0,4$ & $50 \%$ & $37 \%$ & $37 \%$ & $6 \%$ \\
\hline
\end{tabular}

Tableau 3. - Ecart relatif entre la position de $P_{n}(\max )$ théorique et numérique.

Table 3. - Relatif deviation between theoretical and numerical value of $P_{n}$ (max).

\begin{tabular}{|l|c|c|c|c|c|}
\hline \multirow{2}{*}{ Trémies } & \multicolumn{4}{|c|}{ Modèles analytiques } \\
\cline { 2 - 6 } & Matériaux & MOTZKUS & DABROWSKI & LENCZNER & WALKER \\
\hline \multirow{3}{*}{ Trémie $70^{\circ}$} & sable dense $\mu=0,5$ & $-62 \%$ & $51 \%$ & $112 \%$ & $60 \%$ \\
\cline { 2 - 7 } & blé $\mu=0,5$ & $4 \%$ & $107 \%$ & $117 \%$ & $19 \%$ \\
\hline \multirow{3}{*}{ Trémie $75^{\circ}$} & sable meuble $\mu=0,25$ & $-69 \%$ & $22 \%$ & $139 \%$ & $144 \%$ \\
\cline { 2 - 7 } & sable meuble $=0,4$ & $32 \%$ & $-24 \%$ & $34 \%$ & $23 \%$ \\
\cline { 2 - 7 } & blé $\mu=0,25$ & $-30 \%$ & $189 \%$ & $133 \%$ & $118 \%$ \\
\cline { 2 - 7 } & blé $\mu=0,4$ & $-4 \%$ & $-71 \%$ & $161 \%$ & $98 \%$ \\
\hline
\end{tabular}

Tableau 4 - Ecart relatif entre $P_{v}(\max )$ théorique et numérique.

Table 4 - Relatif deviation between theoretical and numerical value of $P_{v}(m a x)$.

\begin{tabular}{|c|c|c|c|c|c|}
\hline \multirow{2}{*}{ Trémies } & \multicolumn{3}{|c|}{ Modèles analytiques } \\
\cline { 2 - 6 } & Matériaux & MOTZKUS & DABROWSKI & LENCZNER & WALKER \\
\hline \multirow{3}{*}{ Trémie $70^{\circ}$} & sable dense $\mu=0,5$ & $-3 \%$ & $28 \%$ & $27 \%$ & $60 \%$ \\
\cline { 2 - 7 } & blé $\mu=0,5$ & $13 \%$ & $46 \%$ & $40 \%$ & $19 \%$ \\
\hline \multirow{3}{*}{ Trémie $75^{\circ}$} & sable meuble $\mu=0,25$ & $7 \%$ & $15 \%$ & $49 \%$ & $144 \%$ \\
\cline { 2 - 7 } & sable meuble $=0,4$ & $-4 \%$ & $-55 \%$ & $35 \%$ & $23 \%$ \\
\cline { 2 - 7 } & blé $\mu=0,25$ & $33 \%$ & $9 \%$ & $33 \%$ & $118 \%$ \\
\cline { 2 - 7 } & blé $\mu=0,4$ & $35 \%$ & $-4 \%$ & $36 \%$ & $98 \%$ \\
\hline
\end{tabular}

obtenues par le programme de simulation, sauf pour le maximum de $\mathrm{P}_{\mathrm{n}}\left(\mathrm{P}_{\mathrm{n}}(\max )\right)$. Les résultats de la norme P 22-630 (norme française) sont proches des valeurs numériques obtenues.

\section{CONCLUSION}

La simulation des charges dans les trémies de silos montre l'influence très significative des paramètres géo- métrique et mécanique de la trémie sur la distribution des pressions. La variation du coefficient de frottement en paroi engendre des courbes de pression d'allures identiques, par contre l'inclinaison de la paroi fait varier la position du maximum de pression. Cette position de Pmax varie linéairement en fonction de l'inclinaison $\alpha$. La technique de simulation du remplissage (en une seule étape et en dix étapes) carac- 


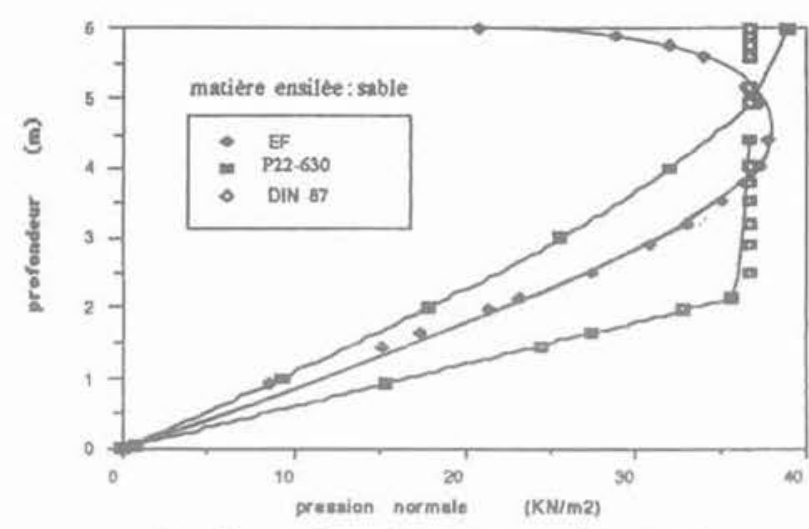

Fig. 32. - Contrainte normale à la paroí. DIN 87 - P22-630 - calcul par EF.

Fig. 32. - Normal wall stresses.

DIN 87 - P22-630. FE method

térise l'influence de la mise en place de la matière sur l'évolution des pressions. La simulation montre également les limites du comportement type " silo " en faisant varier l'élancement. Il ressort de l'étude comparative une non concordance des résultats numériques et analytiques. En effet, les théories classiques, basées sur le calcul des contraintes dans la partie cylindrique du silo, ne sont pas adaptées aux trémies.

\section{REMERCIEMENTS}

Cette étude est réalisée au laboratoire de Structures de l'Université de Karlsruhe, nous tenons à remercier vivement MM. EIBL professeur doct. ing,, ROM$\mathrm{BACH}$ doct. ing. et RUCKENBROD dipl. ing. pour leur aide efficace.

\section{BIBLIOGRAPHIE}

(1) REMESCH J.C., KHELIL A., ROTH J.C. (1990), Simulation numérique de contraintes au sein de matières granulaires ensilées. Comparaison avec les mesures in situ. Rev. Franç. Géotech., $\mathrm{n}^{\circ}$ 52, pp. 51-65.

(2) EIBL J., ROMBACH G. (1987), Numerical computation of velocity fields in silos, theory and application,. Proceedings silosy, Sklarska, Poreba, october 1987.

(3) WEIDNER J. (1990), Vergleich von Stoffgesetzen granularer Schüttgüter zur Silodruckermittlung. Dissertation Karlsruhe 1990.

(4) MOTZKUS U. (1973), Belastung von Siloböden und Auslauftrichtern durch körnige Schüttgüter. Dissertation Braunschweig 1973.

(5) DABROWSKI R. (1989), Zum statischen Silodruck im Kreizylinder mit Kegeltrichter oder Kegelboden. Bautechnik 66, 1989. 